\title{
Simulating the hydrodynamic response of a floater-net system in current and waves
}

\author{
Chen, Hao; Christensen, Erik Damgaard
}

Published in:

Journal of Fluids and Structures

Link to article, DOI:

10.1016/j.jluidstructs.2018.01.010

Publication date:

2018

Document Version

Peer reviewed version

Link back to DTU Orbit

Citation (APA):

Chen, H., \& Christensen, E. D. (2018). Simulating the hydrodynamic response of a floater-net system in current and waves. Journal of Fluids and Structures, 79, 50-75. https://doi.org/10.1016/j.jfluidstructs.2018.01.010

\section{General rights}

Copyright and moral rights for the publications made accessible in the public portal are retained by the authors and/or other copyright owners and it is a condition of accessing publications that users recognise and abide by the legal requirements associated with these rights.

- Users may download and print one copy of any publication from the public portal for the purpose of private study or research.

- You may not further distribute the material or use it for any profit-making activity or commercial gain

- You may freely distribute the URL identifying the publication in the public portal

If you believe that this document breaches copyright please contact us providing details, and we will remove access to the work immediately and investigate your claim. 


\title{
Simulating the hydrodynamic response of a floater-net system in current and waves
}

\author{
Hao Chen ${ }^{\mathrm{a}, *}$, Erik Damgaard Christensen ${ }^{\mathrm{a}}$ \\ ${ }^{a}$ Section of Fluid Mechanics, Coastal and Maritime Engineering, Department of Mechanical Engineering, Technical \\ University of Denmark, DK-2800 Kgs. Lyngby, Denmark
}

\begin{abstract}
We present a novel numerical model for simulating current and wave interaction with a floater-net system. The main contribution of the paper is the integration of the floater motion and the fluid-structure interaction analysis of the net structure in the same modelling framework via the computational fluid dynamic approach. The sinker and the mooring lines were not directly resolved, but their effects were partially modelled. The model couples a hydrodynamic solver, a rigid body motion solver, a mesh motion solver and a structural solver in a segregated manner. In the numerical model, the net structure was modelled as a set of dynamic porous zones. A lumped mass model was coupled with it to realize fluid-structure interaction analysis for the net structure. The floater was treated as a rigid body, which was resolved by the body-fitted computational mesh in the fluid domain. The motion equation for the floater was set up based on the principle of linear and angular momentum balance. Different motion integration schemes were implemented and tested in the numerical model. The numerical model was validated against three sets of available experimental data in the open literature. The first set of validation cases treated the floater motion in regular waves. The second set of validation cases focused on the fluid-structure interaction analysis of the net structure. The final one was related to the whole floater-net system in regular waves, and combined current and wave condition.
\end{abstract}

Keywords: floater-net system, floating body, fluid-structure interaction, OpenFOAM

\section{Introduction}

Hydrodynamic analysis on the floating fish cages for offshore aquaculture has been presented during the past few years. This comes with the fast development of aquaculture industry in the world. With the growing demand of food due to increasing population over the world, aquaculture is expected to continue making important contributions on world food security and nutrition supply.

The fish cage itself is a compliant and flexible system, whose components interact with each other. It usually contains four important components, namely the net cage, the floater, the mooring system and the sinker system. Each of them plays an important role and has its unique functionality. It is in general not straightforward to perform global analysis on the floating fish cage system. Some simplifications and assumptions are needed, in order to set up a realistic numerical model. For instance, there exist a large number of twines and knots for a net cage, which are usually grouped together into fewer meshes in the numerical model. The floater may contain several tori, but it is usually simplified into a circular cross section in the numerical model. However, on the other side one still needs to properly take the effects of all the components into account, although sometimes it may be not necessary to model all of them.

There have been several publications on dynamic analysis of floating fish cage system in current and waves in two-dimension, both numerically and experimentally. Fu et al. (2014) conducted series of experiments to investigate the roles of the net panel and the floater in steady and oscillatory flow. In their experiments, the

\footnotetext{
*Corresponding author, tel.: (45) 50302416; e-mail address: cfd_hchen@outlook.com; fax (45) 45251961.
} 
$\langle\boldsymbol{u}\rangle \quad$ volume averaged velocity field

$\left\langle p_{d}\right\rangle^{f} \quad$ intrinsic volume averaged pore pressure field

$\mu \quad$ dynamic viscosity

$\omega_{w} \quad$ wave circular frequency

$\rho \quad$ density field

$C \quad$ quadratic porous resistance coefficients

$C_{n}^{m} \quad$ added mass coefficient of the net

$C_{n}^{\text {twine }}$ drag force coefficient of the twines of the net

$C_{s}^{d} \quad$ drag force coefficient of the sinker

$C_{s}^{L} \quad$ lift force coefficient of the sinker

$C_{s}^{M} \quad$ inertia coefficient of the sinker, which equals to $C_{s}^{m}+1$

$C_{s}^{m} \quad$ added mass coefficient of the sinker

$D_{s} \quad$ diameter of the sinker

$d_{\text {twine }}$ diameter of the twine of the net

$f_{w} \quad$ wave frequency

$H_{w} \quad$ wave height

$k_{w} \quad$ wave number

$L_{s} \quad$ length of the sinker

$m_{f} \quad$ mass of the floater

$m_{f, a}$ added mass of the floater

$n \quad$ porosity of the net

$p \quad$ total pressure field

$p_{d} \quad$ excess pressure field

$S_{n} \quad$ solidity ratio of the net cage

$T_{w} \quad$ wave period

$U_{c} \quad$ magnitude of steady current velocity

$U_{s} \quad$ magnitude of horizontal sinker velocity

$U_{w} \quad$ magnitude of horizontal wave velocity

$V_{P} \quad$ volume of the porous zones

$V_{s} \quad$ magnitude of vertical sinker velocity

$V_{w} \quad$ magnitude of vertical wave velocity

$W_{s} \quad$ mass of the sinker 
wave condition was realized by forced oscillation of the system. Therefore, the effects of vertical motion of water particles were neglected. The magnitude of the forces on the floater-net system under these conditions were measured and analysed. Based on the experimental data, a hybrid empirical-numerical method was proposed in Ma et al. (2016). In Bardestani and Faltinsen (2013), waves were generated in a narrow wave tank to investigate the interaction between the net, floater and sinker system. Meanwhile, the numerical model was also developed in their work. The force model they applied was based on the screen type force model developed in Kristiansen and Faltinsen (2012). A time domain potential code was used for computation of floater motion, where a convolution integral term was used in the equation to account for the memory effects. In their experiments, it was found that due to the relative motion between the sinker and the floater, very high snap load was observed under regular wave condition with certain wave periods and heights. It lasted very short time, but the magnitudes were several times larger than the static load. Their numerical model could also reproduce this phenomenon. In addition, the mooring line forces in combined current and wave conditions were also measured in the experiments and predicted by the numerical model.

Three-dimensional computation on the model scale fish cage has also been presented in the previous works, but with different level of simplifications. Zhao et al. (2007) presented a numerical study where the floater, net and bottom sinker were all included. The floater was treated as a rigid body, and six degree of freedom motion was considered, where the hydrodynamic force on the floater was calculated based on Morison type force model. The net cage was represented by a lumped mass model, and the forces on the net were also calculated based on Morison equation. Xu et al. (2013) and Xu et al. (2014) applied a similar model. Further improvement was introduced in Zhao et al. (2015), where a curved beam model was used for structural response of the floater. Modal superposition method was introduced to express the deformation of the floater as a weighted sum of eigenmodes. Kristiansen and Faltinsen (2015) extended his previous developed screen type force model in Kristiansen and Faltinsen (2012), where other important components of the fish cage system were included in the numerical model. A truss model was applied to model the net cage deformation, where a linear system of equations for the truss tensions were solved at each time step. The hydrodynamic forces on the floater not only include viscous force and Froude-Kriloff force, but also diffraction and radiation force. A variety of experiments were also conducted in Kristiansen and Faltinsen (2015) to provide benchmark data for the numerical model.

With the increasing computer resources which enhance the ability to handle nonlinear equations, the computational fluid dynamic (CFD) approach is progressively gaining attention in coastal and offshore hydrodynamic community over the past decades. From the recent publications, CFD approach has also been applied to model flow through fixed fishing nets as shown in Patursson et al. (2010) and Zhao et al. (2013), where the net was modelled as a sheet of porous media. Therefore, the geometry of the net cage at pore scale was not resolved, but the effects of the net were represented by a resistance term. In Chen and Christensen (2016), this term was formulated based on the Morison type force model. In addition, due to the flexibility of the net, the effects of the structural deformation should also be taken into account in the numerical model. Attempts have been made to couple the lumped mass model with the porous media model to achieve fluid-structure interaction (FSI) analysis in Yao et al. (2016), Bi et al. (2014b) and Bi et al. (2014a), under steady current condition. Chen and Christensen (2017) further extended the work in Bi et al. (2014b) and Bi et al. (2014a), which made the model applicable in both steady and unsteady conditions.

In general, under steady current condition, the net cage is the main component to stand the current load. The floater has a displacement effect on the flow field, where the streamlines close to the free surface were altered by the floater. Meanwhile, a boundary layer was formed in the vicinity of the floater. However, under wave condition, it is important to consider the floater motion, which is the main contributor to the forces on the net (Lader and Fredheim, 2006). As mentioned above, due to relative motion between the floater and the sinker, the net may get slack and very high snap load may occur.

Therefore, in the present work we will further develop our CFD model presented in Chen and Christensen (2016) and Chen and Christensen (2017). The capability of the numerical model will be extended, where a six degree of freedom motion solver is incorporated in the numerical model. Hereby it is able to model the motion of the floater and the deformation of the net simultaneously via the CFD approach. This is also the main contribution of the paper. Comparing with the existing approaches, application of this CFD model for dynamic analysis of the fish cage system has several advances: (1) The flow field around both the 
floater and the net can be modelled in the numerical model, where no empirical coefficients are needed to predict the flow in the wake. (2) For flow around the floater, the inertia effect is dominant while the viscous effect is minor. On the other hand, regarding the flow around net cage, it is vise versa. The CFD approach is a direct application of the first principles, namely Navier-Stokes equations. Therefore, by application of this approach, both inertia and viscous effects of the fluid flow are naturally accounted for in the numerical model. (3) The nonlinearity of the free surface flow is treated in the numerical model. Therefore, the numerical model is capable of modelling nonlinear waves and its interaction with the floating floater.

The remainder of the paper is organized as follows. In Section 2 a detailed description of the numerical model is given, which includes an overview of the numerical model in Section 2.1. a description of the hydrodynamic model for the floater and the net cage in Section 2.2. a description of the rigid body motion model for the floater in Section 2.3. a description of the mesh motion model in Section 2.4 and a description of the structural model for the net cage and its coupling with the floater motion model and the hydrodynamic model in Section 2.5. Then the numerical model is carefully validated against the experimental data in the open literature in Section 3. It should be mentioned that as the first step to develop the CFD model for floater-net system, the present paper restricts the validation to two dimensional cases. The first set of validation cases concern with the motion of the floater in regular wave conditions, which aims to examine the performance of floater motion solver in two-dimensional cases. The second set of validation cases are focused on the plane net panel in steady current, which demonstrates the applicability of the FSI solver for the net. Then the final validation cases move to the global analysis of the floater-net system, where the snap load on the net panel and the mooring line forces are compared with the experimental data. Finally, conclusions are given in Section 4 .

\section{Description of the numerical model}

\subsection{Overview of the numerical model}

In the present numerical model, we mainly focus on modelling the floater-net system for a fish cage. The modelling framework was the open source toolbox OpenFOAM, and the version 3.0.x was utilized. Here .x means bug-fixed version. This version introduced some new features on the rigid body motion solver, hereby it was preferred and applied. A body-fitted mesh was applied in the numerical model, where the floater was resolved by the mesh, i.e. the floater was treated as a boundary patch in the fluid domain. A six degree of freedom motion solver was applied to obtain the motion status of the floater at each time step. The net structure was modelled as a sheet of porous media, and it was coupled with a lumped mass structural model. The advantage of the applied coupling scheme is that, the hydrodynamic solver for the net did not require the mesh to conform the deformed geometry of the net. This means that the mesh only deformed according to the motion of the floater. Then the porous media zones representing the net structure were updated based on the nodal position of the deformed net. The relative information was transferred between the floater and the net by the mutual mass points that were attached in both the net and the floater.

\subsubsection{Assumptions and simplifications}

Presently in our numerical model, two main simplifications were made. The first simplification was that the mooring lines were not directly resolved. Instead, the restoring forces from the mooring lines were added into the motion equation for the floater. There exist different choices for the force-elongation relation. However, we mainly used the simple linear relation, i.e. the mooring lines were simplified as linear springs. Therefore, the mooring line dynamics and its coupling with the floater were not considered in the numerical model.

In addition, the sinker of the fish cage was also neglected in the numerical model. Only the constant forces on the sinker were added to the bottom mass points of the net. In principle, the sinker was subjected to gravity force, buoyancy force, the force from the net cage and the hydrodynamic force. The gravity and buoyancy force were always included, irrespective of the different cases. However, regarding hydrodynamic force, the force components that were considered in current or wave cases were different. In steady current 
condition, the hydrodynamic force on the sinker was estimated as:

$$
\begin{aligned}
& F_{s, x}^{c u r}=\frac{1}{2} \rho C_{s}^{d} D_{s} U_{c}^{2} L_{s} \\
& F_{s, y}^{c u r}=\frac{1}{2} \rho C_{s}^{L} D_{s} U_{c}^{2} L_{s}
\end{aligned}
$$

The drag and lift force coefficient, namely $C_{s}^{d}$ and $C_{s}^{L}$ varies periodically with time due to vortex shedding. However, the mean drag force coefficient was not zero and it was accounted for in the numerical model. Meanwhile, the mean lift force coefficient vanished. The transient transverse lift force was not considered in the numerical model.

However, in wave cases, the situation becomes more complex. Both the wave motion and the motion of the sinker should be considered. The hydrodynamic force on the sinker in wave cases could be estimated by the modified Morison equation:

$$
\begin{aligned}
& F_{s, x}^{\text {wave }}=\frac{1}{2} \rho C_{s}^{d} L_{s} D_{s}\left(U_{w}-U_{s}\right)\left[\left(U_{w}-U_{s}\right)^{2}+\left(V_{w}-V_{s}\right)^{2}\right]^{1 / 2}+\frac{D_{s}^{2}}{4} \rho L_{s} C_{s}^{M} \pi \frac{\partial U_{w}}{\partial t}-\frac{D_{s}^{2}}{4} \rho L_{s} C_{s}^{m} \pi \frac{\partial U_{s}}{\partial t} \\
& F_{s, y}^{\text {wave }}=\frac{1}{2} \rho C_{s}^{L} L_{s} D_{s}\left(V_{w}-V_{s}\right)\left[\left(U_{w}-U_{s}\right)^{2}+\left(V_{w}-V_{s}\right)^{2}\right]^{1 / 2}+\frac{D_{s}^{2}}{4} \rho L_{s} C_{s}^{M} \pi \frac{\partial V_{w}}{\partial t}-\frac{D_{s}^{2}}{4} \rho L_{s} C_{s}^{m} \pi \frac{\partial V_{s}}{\partial t}
\end{aligned}
$$

Presently in the numerical model, $F_{s, x}^{\text {wave }}$ and $F_{s, y}^{\text {wave }}$ were neglected.

In general, the benefit of this simplification is that, the whole model was simplified from a multi-body problem to a single body problem. Therefore, it greatly eased the manipulation of the mesh. The mesh quality for a single body problem is usually much better than for a multi-body problem, especially considering the amplitude of motion of the sinker. In large steady current flow, the movement of the sinker may easily distort the mesh. However, the price we paid was that, the unsteady load on the sinker and its interaction effects with the net structure were not considered. In steady current condition, the influence of this assumption should be insignificant, since the mean drag force was usually the dominating part. The oscillating drag and lift force have minor effects. But when it comes to the wave cases, this simplification might be questionable in some cases. At the given depth where the sinker was located, the orbital motion of the water wave particle became rather weak. Hereby the second term in Eq. (3) and Eq. (4) have rather limited influences. But the first and third term were dependent on the relative velocity rather than the absolute water particle velocity. Neglecting of these two terms serve as an important error source of the numerical model. In Section 3.3 further error analysis will be given on this issue.

\subsubsection{Flowchart of the algorithm}

The numerical model was extended based on the solver developed in Jacobsen et al. (2012), which is a multiphase solver for two incompressible, isothermal and immiscible fluids with the functionality on generation and absorption of gravity water waves. In the present work, it was coupled with the rigid body motion solver, the mesh motion solver, and the structural solver for the net in a segregated manner. A flow chart of the solver is given in Fig. 1 .

For modelling of the floater motion in waves, one should be careful on the so-called artificial added mass effect. This is due to the fact that the densities of the floater and the water are usually in the same order of magnitude. Therefore, part of the fluid may act as an extra mass on the structural degrees of freedom at the coupling interface. In sequentially staggered schemes the fluid forces depend upon the predicted displacement of the floater rather than the corrected ones, which contain a portion of incorrect coupling forces. It is this artificial contribution to the coupling which yields the instability (Förster et al., 2007). Therefore, in order to handle this issue properly, we introduced the concept of sub-iteration within one time step in the numerical model, which converges the computed quantities.

To begin with a sub-iteration, the forces on the floater were updated first by adding the gravity force, the updated wave force, mooring line force and the force from the net structure. Then the linear acceleration 


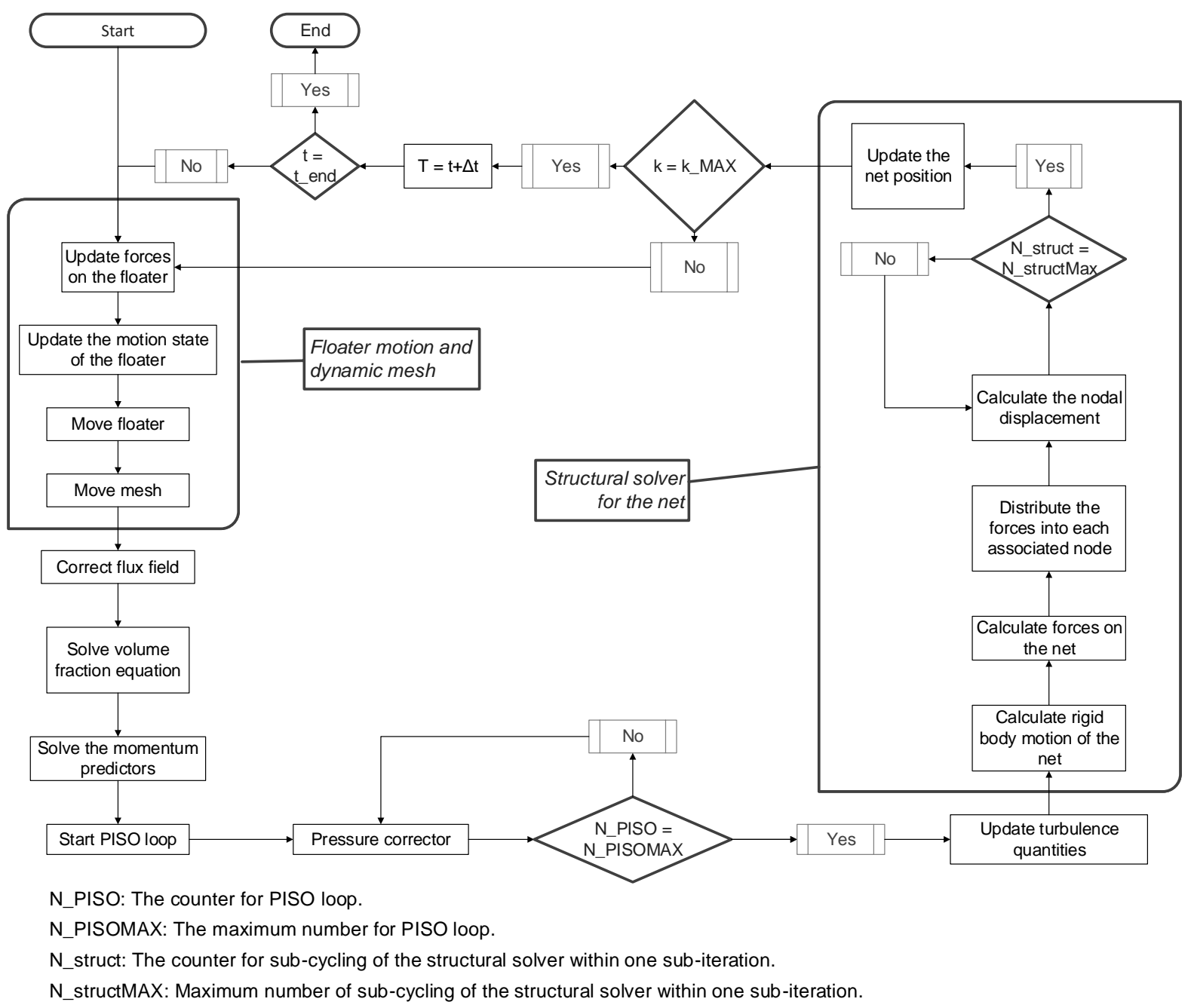

Figure 1: Flow chart of the custom solver.

$\boldsymbol{a}_{f}$ and the torque $\boldsymbol{\tau}_{f}$ were obtained based on the force and momentum balance equations. Given $\boldsymbol{a}_{f}$ and $\boldsymbol{\tau}_{f}$, the linear velocity $\boldsymbol{u}_{f}$, angular momentum $\boldsymbol{L}_{f}$, position of center of mass $\boldsymbol{x}_{f}$ and altitude $\boldsymbol{Q}_{f}$ were calculated by choosing a proper integration method. Then the boundary patch of the floater was moved, and the mesh was deformed based on the calculated motion status. Following the dynamic mesh motion solver, the flow solver solved Navier-Stokes equations for a mixture of water and air phases. Specifically, in the porous media region, the Navier-Stokes equations were volume averaged to consider the effect of net structure on the fluid. With the updated pressure and velocity field, the hydrodynamic forces on the net structure were determined. A lumped mass structural model was coupled with the hydrodynamic model to resolve its deformation. This forms a closed sub-iteration.

From our practical experience, in many cases with small to medium wave amplitudes, a loosely coupled algorithm is still capable and can produce results with satisfying accuracy. But when the wave height is increasing, which leads to large amplitude of floater motion, sub-iterations are necessary. By convergence test, it was found that $4-8$ sub-iterations within one time step can produce satisfactory results. Too many sub-iterations do not necessarily improve the simulation results. Below from Section 2.2 to 2.5 , a thorough introduction on each part of the numerical model is given, with illustrations on how it is coupled with other solvers. 


\subsection{Hydrodynamic model}

\subsubsection{Hydrodynamic model for the floater}

The governing equations in the fluid domain except the net region are the mass and momentum conservation for two incompressible, isothermal and immiscible fluids:

$$
\begin{gathered}
\nabla \cdot \boldsymbol{u}=0 \\
\frac{\partial \rho \boldsymbol{u}}{\partial t}+\nabla \cdot \rho \boldsymbol{u} \boldsymbol{u}_{c}-\nabla \cdot \mu \nabla \boldsymbol{u}=-\nabla p_{d}-(\boldsymbol{g} \cdot \boldsymbol{x}) \nabla \rho
\end{gathered}
$$

Due to application of moving mesh technique, the relative velocity $\boldsymbol{u}_{c}$ was applied as the convective velocity field, which allows the physical quantities to be described in arbitrary Lagrangian-Euler formulation. $\boldsymbol{u}_{c}$ itself is not necessary to be divergence free. However, it was constrained to obey the space conservation law, in case any mass conservation error was introduced. In addition, the excess pressure field $p_{d}$ was applied in Eq. (6), and the total pressure field was reconstructed at every time step as:

$$
p=p_{d}+\rho \boldsymbol{g} \cdot \boldsymbol{x}
$$

In order to handle the generation and propagation of free surface waves, a free surface tracking method needs to be applied. Volume of fluid (VOF) method was applied in the present model, and a transport equation for the volume fraction field was solved:

$$
\frac{\partial \alpha}{\partial t}+\nabla \cdot \boldsymbol{u}_{c} \alpha+\nabla \cdot\left(\boldsymbol{u}_{r} \alpha(1-\alpha)\right)=0
$$

Eq. (8) is a modified version of the original scalar transport equation, e.g. as shown in Hirt and Nichols (1981). An extra term was introduced to sharpen the free surface. The formulation itself was inherited from the two-fluid model, where $\boldsymbol{u}_{r}$ was denoted as the relative velocity between two fluids. However in volume of fluid method, the slip velocity does vanish. $\boldsymbol{u}_{r}$ was retained solely to numerically compress the free surface, and it is only active in the free surface region, due to the multiplication of $\alpha(1-\alpha)$.

\subsubsection{Hydrodynamic model for the net}

The porous media model was applied to describe flow through the net structure, where it was represented by a sheet of porous media zone. Jensen et al. (2014) revised the formulation for porous media model based on the volume averaged Navier-Stokes equations, which has been applied in our previous works in Chen and Christensen (2016) and Chen and Christensen (2017). However, in the present work, again due to mesh motion, the convective velocity $\boldsymbol{u}_{c}$ should be applied:

$$
\begin{gathered}
\nabla \cdot\langle\boldsymbol{u}\rangle=0 \\
\left(1+C_{n}^{m}\right) \frac{1}{n} \frac{\partial \rho\langle\boldsymbol{u}\rangle}{\partial t}+\frac{1}{n} \nabla \cdot \frac{\rho}{n}\langle\boldsymbol{u}\rangle\left\langle\boldsymbol{u}_{c}\right\rangle=-\nabla\left\langle p_{d}\right\rangle^{f}-\boldsymbol{g} \cdot \boldsymbol{x} \nabla \rho+\frac{1}{n} \nabla \cdot \mu \nabla\langle\boldsymbol{u}\rangle+\boldsymbol{S}
\end{gathered}
$$

In Eq. (10), due to the averaging process, several new quantities were introduced. The coefficient $C_{n}^{m}$ represents the added mass effect on the porous skeleton, and was expressed as:

$$
C_{n}^{m}=\gamma_{p} \frac{1-n}{n}
$$

where $\gamma_{p}$ is an empirical coefficient, and takes the value of 0.34 (See Chen and Christensen (2016) for the explanation). The resistance force used in Eq. (10) was expressed as:

$$
\boldsymbol{S}=-\frac{1}{2} \rho C\left|\boldsymbol{u}-\boldsymbol{u}_{\boldsymbol{n}}\right|\left(\boldsymbol{u}-\boldsymbol{u}_{\boldsymbol{n}}\right)
$$


Eq. (12) uses the relative velocity for calculation of the resistance, due to the relative motion between the fluid and the net. By application of Eq. (12), both the drag force and the transverse lift force exerted on the net structure are considered. $C$ is the quadratic porous resistance coefficient matrix. In its local coordinate it is given as:

$$
C=\left[\begin{array}{ccc}
C_{1} & 0 & 0 \\
0 & C_{2} & 0 \\
0 & 0 & C_{3}
\end{array}\right]
$$

The formula proposed in Chen and Christensen (2016) was applied in the present work to relate the magnitude of $C_{1}, C_{2}$ and $C_{3}$ with the physical parameters of the net cage. Regarding the detailed derivation process, readers are referred to that paper. Here we briefly recapitulate the basic process. The first step is to obtain the coefficients $a$ and $b$, which empirically accounts for the interaction effects in-between the twines. They were functions of the solidity ratio $S_{n}$ of the given net, and the expressions were given as:

$$
\begin{gathered}
a=\left\{\begin{array}{lc}
2.3484 S_{n}+1 & 0<S_{n} \leq 0.13 \\
1.3128 S_{n}+1.1346 & 0.13<S_{n} \leq 0.243 \\
5.3094 S_{n}+0.1634 & 0.243<S_{n} \leq 0.317
\end{array}\right. \\
b=\left\{\begin{array}{lc}
0.9241 & 0<S_{n} \leq 0.13 \\
-0.6310 S_{n}+1.0061 & 0.13<S_{n} \leq 0.243 \\
8.7581 S_{n}-1.2754 & 0.243<S_{n} \leq 0.317
\end{array}\right.
\end{gathered}
$$

Then the porous coefficients $C_{1}, C_{2}$ and $C_{3}$ were calculated by

$$
\begin{aligned}
C_{1} & =\frac{1}{V_{P}} a C_{n}^{\text {twine }}\left(S_{1}+S_{2}\right) \\
C_{2} & =\frac{1}{V_{P}} b C_{n}^{\text {twine }} S_{2} \\
C_{3} & =\frac{1}{V_{P}} b C_{n}^{\text {twine }} S_{1}
\end{aligned}
$$

where $S_{1}$ is the total projected area for in-plane twines and $S_{2}$ is the total projected area for out-of-plane twines. The definitions of $S_{1}$ and $S_{2}$ have been given in Fig. 1 and Fig. 2 in Chen and Christensen (2016). In addition, $V_{P}$ is the volume of the porous zones, and $C_{n}^{\text {twine }}$ is the drag force coefficient of the twines.

Eq. (8) was also revised to include the porosity effect:

$$
\frac{\partial \alpha}{\partial t}+\frac{1}{n} \nabla \cdot \boldsymbol{u}_{c} \alpha+\frac{1}{n} \nabla \cdot \boldsymbol{u}_{r} \alpha(1-\alpha)=0
$$

The correction by the factor $1 / \mathrm{n}$ ensures that it is only the pore volume, instead of the cell volume, that can be filled with water. With the reduced volume in the porous zones, the cell has to be filled/emptied faster. On the other hand, the upper limit of $\alpha$ has to be one in that cell. So that the fluid properties can still be calculated as a weighted average.

\subsection{Rigid body motion model for the floater}

A rigid body motion solver was applied to obtain the motion status of the floater, which includes the altitude $\boldsymbol{Q}_{f}$, the linear velocity $\boldsymbol{u}_{f}$, the linear acceleration $\boldsymbol{a}_{f}$, the torque $\boldsymbol{\tau}_{f}$, the angular momentum $\boldsymbol{L}_{f}$ and the position of center of mass of the floater $\boldsymbol{x}_{f}$. However, below for simplicity, we only focus on the linear quantities, namely $\boldsymbol{a}_{f}, \boldsymbol{u}_{f}$ and $\boldsymbol{x}_{f}$. But the angular quantities in principle are obtained in the same way. It should be mentioned that the momentum balance was evaluated in the local body reference which was aligned with the principle axis of the inertia and with origin at the center of mass. This is advantageous since the moment of inertia was not changing in that coordinate system. Transformation of the quantities 
between local and global reference was via the altitude tensor of the body $\boldsymbol{Q}_{f}$. For instance, the torque was transformed to the local body reference before calculating the angular momentum as:

$$
\boldsymbol{\tau}_{f, \text { local }}=\boldsymbol{Q}_{f}^{T} \cdot \boldsymbol{\tau}_{f}
$$

For linear motion of the floater, the governing equation is the Newton's second law:

$$
m_{f} \boldsymbol{a}_{f}=\boldsymbol{F}_{f}^{\text {wave }}+\boldsymbol{F}_{f}^{\text {grav }}+\boldsymbol{F}_{f}^{\text {moor }}+\boldsymbol{F}_{f}^{\text {net }}
$$

where the wave force on the floater is simply the integration of the total pressure and the viscous forces on the floater boundary patch at the instantaneous wet surface at each time step:

$$
\boldsymbol{F}_{f}^{\text {wave }}=-\int_{S_{b}} p \boldsymbol{n}_{b} \mathrm{~d} S-\int_{S_{b}} \boldsymbol{\tau} \cdot \boldsymbol{n}_{b} \mathrm{~d} S
$$

where $S_{b}$ is the boundary surface of the floater, $\boldsymbol{n}_{b}$ is the normal unit vector of the floater surface pointing to the fluid domain. $\tau$ is the viscous stress of the fluid flow. The gravity force was a constant input from the user. The mooring forces were determined by the instantaneous position of the floater and the anchor. The force from the net structure was transferred from the lumped mass model. Then the velocity and center of mass of the floater was obtained by integrating the acceleration at each time step:

$$
\begin{aligned}
& \boldsymbol{u}_{f}=\int \boldsymbol{a}_{f} \mathrm{~d} t \\
& \boldsymbol{x}_{f}=\int \boldsymbol{u}_{f} \mathrm{~d} t
\end{aligned}
$$

There exists different schemes for numerical integration of Eq. 23) and Eq. 24). In the present work we tested four of them, namely the leapfrog scheme, the Newmark scheme, the implicit-explicit AdamsBashforth-Moulton scheme and the Crank-Nicolson scheme. Below we give a brief description on each scheme for the sake of readers to implement the schemes and reproduce the results.

\subsubsection{Leapfrog scheme}

The implementation of the leapfrog scheme follows the way as shown in Dullweber et al. (1997), which consists of three steps to update the motion state:

1. Update the velocity at half new time step (Eq. 25p), and update the position at new time step based on the half time-step velocity (Eq. (26)).

2. Update the force and acceleration at new time step.

3. Update the velocity at new time step (Eq. 277).

$$
\begin{gathered}
\boldsymbol{u}_{f, n}^{0.5}=\boldsymbol{u}_{f, o}+\frac{1}{2} \boldsymbol{a}_{f, o} \Delta t \\
\boldsymbol{x}_{f, n}^{1}=\boldsymbol{x}_{f, o}+\boldsymbol{u}_{f, n}^{0.5} \Delta t \\
\boldsymbol{u}_{f, n}^{1}=\boldsymbol{u}_{f, n}^{0.5}+\frac{1}{2} \boldsymbol{a}_{f, n}^{1} \Delta t
\end{gathered}
$$

Here the subscript $n$ denotes the value at new time step, $o$ denotes the value at old time step. The number at the superscript denotes the number of sub-iteration, e.g. $\boldsymbol{u}_{f, n}^{1}$ is the velocity of the floater at the first sub-iteration at new time step, i.e. the predicted velocity of the floater at new time step.

Leapfrog scheme is fundamentally an explicit scheme as seen from the above description. Therefore, when coupling it with the flow solver, the leapfrog scheme must be applied only once within one time step. This indeed requires that only weakly/loosely coupled algorithm is allowed for this FSI problem, i.e. no implicit 
iterations within one time step. In addition, leapfrog scheme formally is only stable for fixed time step (see e.g. Devolder et al. (2015) and Birdsall and Langdon (2004)). Therefore, if the simulation is performed using variable time step, stability issues may arise due to both reasons. In Section 3.1 when we validated the motion solver, we suffered from some stability problems using this integration method for simulation of floater motion in large amplitude waves. Hereby In Section 3.1 no results are presented using this method.

\subsubsection{Newmark scheme}

The Newmark scheme introduces two parameters $\beta$ and $\gamma$. Based on this scheme, the velocity and position of the body is updated as:

$$
\begin{gathered}
\boldsymbol{u}_{f, n}^{k+1}=\boldsymbol{u}_{f, o}+\Delta t\left(\gamma \boldsymbol{a}_{f, n}^{k}+(1-\gamma) \boldsymbol{a}_{f, o}\right) \\
\boldsymbol{x}_{f, n}^{k+1}=\boldsymbol{x}_{f, o}+\boldsymbol{u}_{f, o} \Delta t+\beta(\Delta t)^{2} \boldsymbol{a}_{f, n}^{k}+(0.5-\beta)(\Delta t)^{2} \boldsymbol{a}_{f, o}
\end{gathered}
$$

We adopted a set of commonly used parameters with $\gamma=0.5$ and $\beta=0.25$, which yields the so-called constant average acceleration method.

\subsubsection{Adams-Bashforth-Moulton scheme}

The implementation of Adams-Bashforth-Moulton scheme follows Seng (2012) and Chow and Ng (2016), which is a predictor-corrector method. It updates the velocity and the position of the floater by the following steps:

1. At the predictor stage, apply Eq. (30) and Eq. (31) for prediction of velocity and displacement.

2. At the corrector stage:

- Update the force and acceleration based on the velocity and displacement from the previous iterations.

- Correct the velocity and displacement based on Eq. (32) and Eq. (33).

- Set $k=k+1$ and repeat the correctors until $k=k_{\max }$.

$$
\begin{gathered}
\boldsymbol{u}_{f, n}^{1}=\boldsymbol{u}_{f, o}+\frac{\Delta t}{2}\left[\left(2+\frac{\Delta t}{\Delta t_{0}}\right) \boldsymbol{a}_{f, o}-\frac{\Delta t}{\Delta t_{0}} \boldsymbol{a}_{f, o o}\right] \\
\boldsymbol{x}_{f, n}^{1}=\boldsymbol{x}_{f, o}+\frac{\Delta t_{0}}{16}\left[\left(1+\frac{8 \Delta t}{\Delta t_{0}}\right) \boldsymbol{u}_{f, n}^{1}+\left(\frac{7 \Delta t}{\Delta t_{0}}-1\right) \boldsymbol{u}_{f, o}+\frac{\Delta t}{\Delta t_{0}} \boldsymbol{u}_{f, o o}\right] \\
\boldsymbol{u}_{f, n}^{k+1}=\boldsymbol{u}_{f, o}+\frac{\Delta t_{0}}{16}\left[\left(1+\frac{8 \Delta t}{\Delta t_{0}}\right) \boldsymbol{a}_{f, n}^{k}+\left(\frac{7 \Delta t}{\Delta t_{0}}-1\right) \boldsymbol{a}_{f, o}+\frac{\Delta t}{\Delta t_{0}} \boldsymbol{a}_{f, o o}\right] \\
\boldsymbol{x}_{f, n}^{k+1}=\boldsymbol{x}_{f, o}+\frac{\Delta t_{0}}{16}\left[\left(1+\frac{8 \Delta t}{\Delta t_{0}}\right) \boldsymbol{u}_{f, n}^{k+1}+\left(\frac{7 \Delta t}{\Delta t_{0}}-1\right) \boldsymbol{u}_{f, o}+\frac{\Delta t}{\Delta t_{0}} \boldsymbol{u}_{f, o o}\right]
\end{gathered}
$$

where the subscript oo denotes the value at the previous two time step.

\subsubsection{Crank-Nicolson scheme}

Crank-Nicolson scheme was introduced as a blending scheme between the explicit and implicit Euler scheme. Two parameters $\omega_{a}$ and $\omega_{u}$ were applied to adjust the blending. The formulation of Crank-Nicolson scheme was given as:

$$
\begin{aligned}
& \boldsymbol{u}_{f, n}=\boldsymbol{v}_{f, o}+\Delta t\left(\omega_{a} \boldsymbol{a}_{f, n}+\left(1-\omega_{a}\right) \boldsymbol{a}_{f, o}\right) \\
& \boldsymbol{x}_{f, n}=\boldsymbol{x}_{f, o}+\Delta t\left(\omega_{u} \boldsymbol{u}_{f, n}+\left(1-\omega_{u}\right) \boldsymbol{u}_{f, o}\right)
\end{aligned}
$$

If $\omega_{a}=0.5$ and $\omega_{u}=0.5$, this method is equivalent to the Newmark integration with $\gamma=0.5$ and $\beta=0.25$. Presently, $\omega_{a}=0.9$ and $\omega_{u}=0.9$ were applied. 
As mentioned above in Section 2.1.2 within each time step, we introduced several sub-iterations. From the second sub-iteration, i.e. the first corrector step, we adopted an under-relaxation factor for the acceleration. Therefore, the acceleration at $k$ th sub-iteration was relaxed as:

$$
\boldsymbol{a}_{f, n}^{k}=\omega_{n}^{k} \boldsymbol{a}_{f, n}^{k}+\left(1-\omega_{n}^{k}\right) \boldsymbol{a}_{f, n}^{k-1}
$$

where $\omega_{n}$ is the under-relaxation factor. The purpose to apply under-relaxation is to enhance the stability of the motion solver. When we compute the acceleration and integrate the motion based on Eq. (21), one part of the force is in phase with the acceleration, namely the added mass force in $\boldsymbol{F}_{f}^{\text {wave }}$. But for a CFD solver, it is usually impossible to separate this part and move it to the left hand of Eq. 211. Hereby this is a source that leads to the instability for the numerical integration. Application of under-relaxation could effectively stabilize the solver.

Proper selection of the relaxation factor is important for the integration schemes. A too high underrelaxation factor may not work robustly, while too low value may slow down the convergence. We applied a dynamic under-relaxation factor as the same in Dunbar et al. (2015), and it was calculated as:

$$
\omega_{n}^{k}=-\omega_{n}^{k-1} \frac{\boldsymbol{r}_{n}^{k-1} \cdot\left(\boldsymbol{r}_{n}^{k}-\boldsymbol{r}_{n}^{k-1}\right)}{\left|\boldsymbol{r}_{n}^{k}-\boldsymbol{r}_{n}^{k-1}\right|^{2}}
$$

where $\boldsymbol{r}^{k}$ was defined as the difference between acceleration of the floater at the current and the previous sub-iteration:

$$
\boldsymbol{r}_{n}^{k}=\boldsymbol{a}_{f, n}^{k}-\boldsymbol{a}_{f, n}^{k-1}
$$

Note that application of Eq. (37) requires the availability of $\boldsymbol{r}^{k}$ and $\boldsymbol{r}^{k-1}$, hereby it was applied from the third sub-iteration. At the second sub-iteration, we applied a fixed under-relaxation factor. Actually Söding (2001) found that the optimal relaxation factor was related to the added mass of the floating body:

$$
\boldsymbol{a}_{f, n}^{k}=\frac{m_{f} \boldsymbol{a}_{f, n}^{k}+m_{f, a} \boldsymbol{a}_{f, n}^{k-1}}{m_{f, a}+m_{f}}=\omega_{n}^{k} \boldsymbol{a}_{f, n}^{k}+\left(1-\omega_{n}^{k}\right) \boldsymbol{a}_{f, n}^{k-1}
$$

which leads to the following expression:

$$
\omega_{n}^{k}=\frac{m_{f}}{m_{f}+m_{f, a}}
$$

Devolder et al. (2015) shows some different convergence behavior by using different relaxation factors when the ratio between the added mass and mass of the floating body was fixed. If Eq. (40) was applied to calculate the under-relaxation factor, the solution was converged very fast. However, in the present work we did not apply Eq. 40, since the added mass is usually not straightforward to estimate and it is frequency dependent. A fixed value of 0.3 was applied in the second sub-iteration for the acceleration of the floater.

\subsection{Mesh motion model}

In the present model we applied the deforming mesh technique. This method has the advantages that it could precisely describe the moving boundary, which means that no interpolation method is needed like immersed boundary method. However, care should be taken when there exists large amplitude of motion for the moving boundaries, especially for rotation motion. Mesh quality can often degrade under such motions. Presently we applied the newly developed mesh morphing technique in OpenFOAM to preserve the mesh quality near the floater.

By application of this method, the computational domain was divided into three regions based on the movement of the cells. These three mesh regions were identified by the distance $d$ from the cell center to the moving boundary. Two values $d^{i}$ and $d^{o}$ were designated by the user in the input file. For each cell, if $d<d^{i}$, then it belongs to the inner region. On the contrast, if $d>d^{o}$, the cell is in the outer region. 


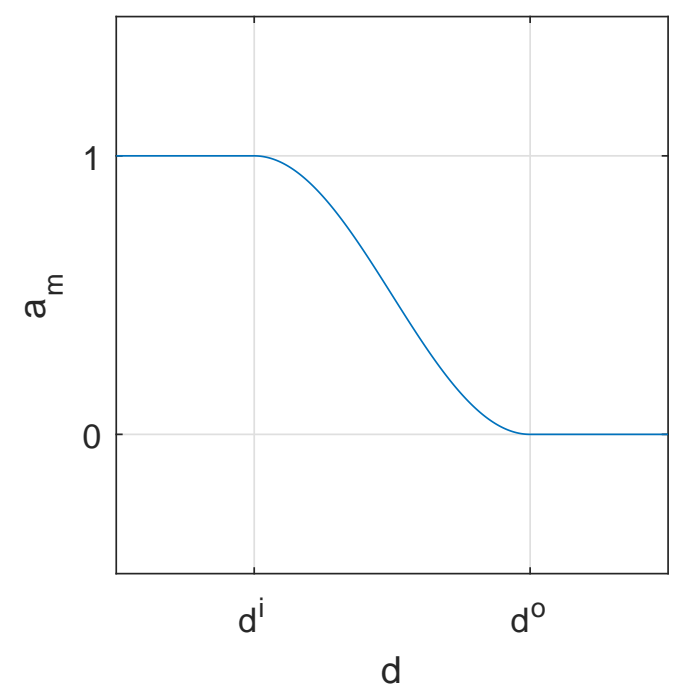

Figure 2: Variation of the motion scale $a_{m}$ as a function of the distance between the cell center and the moving boundary patch $d$.

The rest are in the middle region. The mesh cells in the inner region, which were close to the moving boundary, were moved like a rigid body following the motion of the moving boundary. Hereby the mesh quality near the moving boundary was preserved. In addition, there exists an outer region, where the cells were stationary. Therefore, it is not necessary to move all the cells in the computational domain, which may significantly reduce the computational time when the domain is large. In between the outer region and the inner region, the cells were moved based on the interpolated displacement of the floater motion. In practice, Palm et al. (2016) suggests that $d^{i}$ should be in the order of boundary layer thickness, which is the minimum requirement, since the cells immersed in the boundary layer should be in high quality. Presently we set it equal to the diameter of the floater. $d^{o}$ should be determined based on the motion amplitude of the floater, but it is limited by the minimum distance to any domain boundary.

The spherical linear interpolation (SLERP) method was applied to interpolate the cell displacement and rotation in the middle region. A non-dimensional scale parameter $a_{m}$ was defined here for SLERP method:

$$
a_{m}=\frac{1}{2}-\frac{1}{2} \cos \left(\pi \frac{d^{o}-d}{d^{o}-d^{i}}\right)
$$

It was seen that a cosine profile was applied in this region, as shown in Fig. 2 which plots the value of $a_{m}$ as a function of $d$. This guarantees a smooth transition for the cell displacement and rotation between the outer region and the inner region.

In OpenFOAM, the implementation of SLERP method uses the concept of quaternion to ease the expression for rotation motion. In addition, the translation and rotation were wrapped together as the so-called septernion. Each septernion was composed of a translation vector and a rotation quaternion. The translation vector for a specific cell at the new time step was scaled as:

$$
\boldsymbol{x}_{m, n}^{k}=\boldsymbol{x}_{m, 0}+a_{m, n}^{k}\left(\boldsymbol{x}_{f, n}^{k}-\boldsymbol{x}_{f, 0}\right)
$$

where the subscript 0 denotes the value of the quantity at the initial state. In addition, the rotation quaternion was scaled based on the rotation quaternion of the floater $\boldsymbol{q}_{f, n}$ :

$$
\boldsymbol{q}_{m, n}^{k}=\boldsymbol{q}_{I}\left(\boldsymbol{q}_{I}^{-1} \boldsymbol{q}_{f, n}^{k}\right)^{a_{m, n}^{k}}
$$

where $\boldsymbol{q}_{f, n}$ was transformed from the altitude matrix of the floater at the current time step and at the initial state, i.e. $\boldsymbol{Q}_{f, n}$ and $\boldsymbol{Q}_{f, 0} . \boldsymbol{q}_{I}$ was the unit quaternion defined as $1+0 \boldsymbol{i}+0 \boldsymbol{j}+0 \boldsymbol{k}$. 


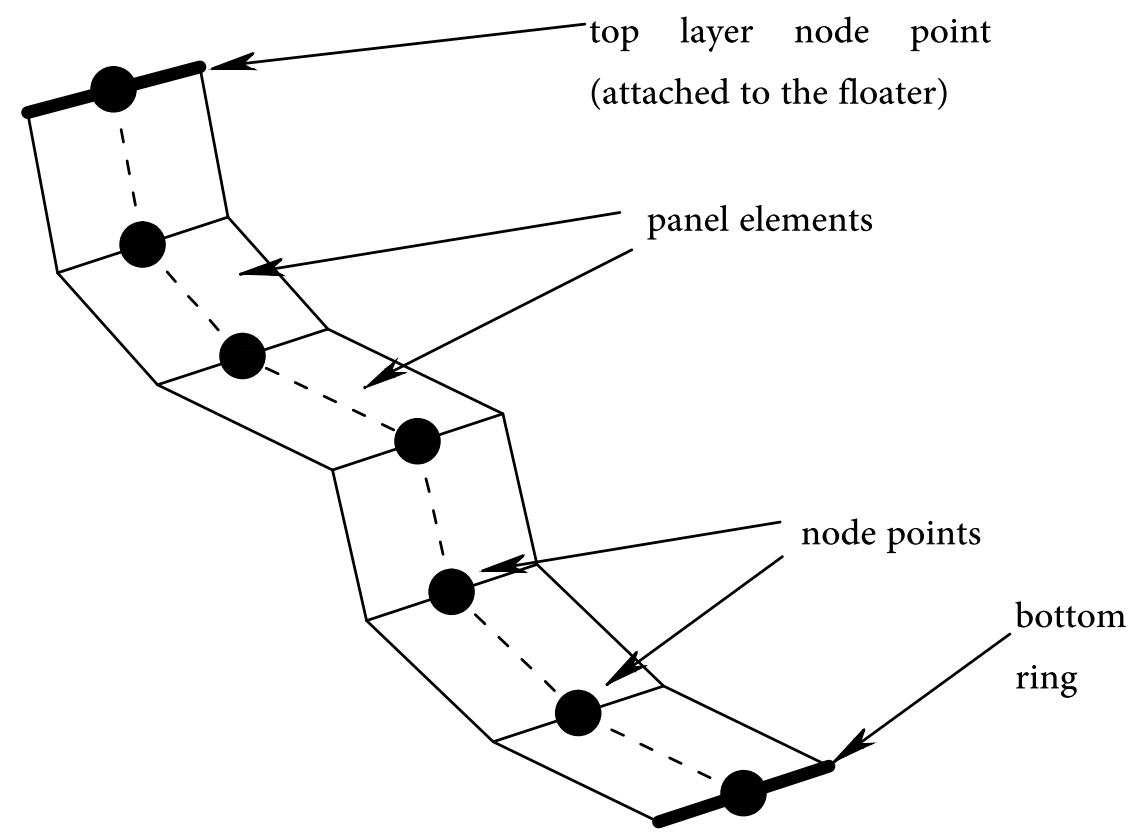

Figure 3: Illustration of the lumped mass structural model in 2D case.

\subsection{Structure model for the net}

\subsubsection{Introduction to the lumped mass model}

In order to resolve the deformation of the net structure at each time step, a lumped mass structural model was implemented and coupled with the hydrodynamic model. The implementation was based on Lader and Fredheim (2006). The idea is to represent the net as a set of nodes and panel elements. In 2D cases the net was vertically divided into panel elements with a node point at the intersection between each element, as shown in Fig. 3. In 3D cases the node points were placed at the element corners.

The forces acting on each node include hydrodynamic force $\boldsymbol{F}_{n}^{\text {wave }}$, structural force $\boldsymbol{F}_{n}^{\text {struct }}$, gravity force $\boldsymbol{F}_{n}^{\text {grav }}$ and buoyancy force $\boldsymbol{F}_{n}^{\text {buoy }} . \boldsymbol{F}_{n}^{\text {grav }}$ and $\boldsymbol{F}_{n}^{\text {buoy }}$ were constant and solely determined by the net properties. Wave force on each panel element was output from the hydrodynamic model based on the flow velocity and the net velocity:

$$
\boldsymbol{F}_{n}^{\text {wave }}=\int_{V_{P}} \frac{1}{2} \rho C\left|\boldsymbol{u}-\boldsymbol{u}_{\boldsymbol{n}}\right|\left(\boldsymbol{u}-\boldsymbol{u}_{\boldsymbol{n}}\right) \mathrm{d} V
$$

This force on the panel was distributed evenly into each node.

The connection between two nodes were modeled as a nonlinear spring. The constitutive relationship of the spring was obtained from experiments as shown in Lader and Fredheim (2006). The relation between the structural force and elongation was expressed as:

$$
F_{n}^{\text {struct }}= \begin{cases}E_{1} \epsilon+E_{2} \epsilon^{2} & \epsilon>0 \\ 0 & \epsilon \leq 0\end{cases}
$$

where $\epsilon$ is the elongation of the spring. Hereby in the numerical model, the net only has negligible compression stiffness. The fitted coefficients are $E_{1}=1160 \mathrm{~N}$ and $E_{2}=37300 \mathrm{~N}$. It should be mentioned that the experiments were conducted for a specific net panel made from Nylon, with a mass density of $1130 \mathrm{~kg} / \mathrm{m}^{3}$. Therefore, there might be variations for these two coefficients for other type of net material. Furthermore, the wet net may have a different constitution relationship from a dry net. And the application of anti-fouling 
techniques on the net may also alter the coefficients. However, in the present work we applied this set of coefficients without further investigations, due to very limited data.

Given the forces on each node, Newton's second law was applied to obtain the acceleration. The linear velocity and the displacement were obtained in the same way with the floater motion, as shown in Eq. 223 and Eq. 241. An explicit Runge-Kutta method provided in OpenFOAM library was applied for the integration.

\subsubsection{Coupling with the hydrodynamic model}

The coupling of the hydrodynamic model with the structural model for the net was based on the concept of dynamic porous zones in the static mesh. This means that in the hydrodynamic model, the net structure was represented by a set of dynamic porous zones corresponding to the panel elements in the structural model. The mesh was not deformed again due to the deformation of the net. Instead at each time step, after moving the mesh due to the motion of the floater, the nodal position was transferred from the structural model to the hydrodynamic model. Then the grid cells in the porous zones were updated based on the transferred nodal positions.

Sub-cycling of the time step for the structural solver was applied, as shown in Fig. 1 where the number of sub-cycling was denoted as $N_{\text {struct }}$. This is due to that the structural solver requires a rather small time step, approximately in the order of $10^{-5} \mathrm{~s}$, while usually in flow solver the time step was around $10^{-3}-10^{-4}$ s. Therefore, the time step in flow solver was set as the global time step. It was assumed that under each global time step, the hydrodynamic load was constant. Under such constant load, the position of the net was evolved.

However, one should note that even with sub-cycling, the time step could not be too large. This is due to two reasons. The first reason is that with very large time step, oscillations were observed on the time series of the hydrodynamic load of the net structure, as shown in Chen and Christensen (2017). The oscillation may further pollute the solution from the motion solver for the floater. Meanwhile, the solution for Eq. (8) also requires a rather small time step, in order to generate the incoming waves with good quality. Hereby we set the maximum Courant number to be around $0.15-0.25$.

\subsubsection{Coupling with the floater motion}

The interaction effects between the floater and the net structure were achieved by utilizing mutual mass points that were attached to both the floater and the net cage, as shown in Fig. 3 for the top layer node point. At each time step, the structural force from the top layer node point was calculated based on Eq. (45), and transferred from the lumped mass model to the floater motion model. After solving Eq. (23) and Eq. (24), the translation and rotation of the floater at the positions of the mass points were also stored. Hereby the structural force on the net and the induced deformation were calculated based on the updated positions of the top layer mass points.

\section{Validation of the numerical model}

In this section, thorough validations were carried out for the above described numerical model. To begin with, the motion solver for the floater was validated against experimental results in Section 3.1. Then the second set of validation cases were on the FSI solver for net structure, as shown in Section 3.2. Finally, Section 3.3 presents validation cases on the floater-net system.

\subsection{Validation of the motion solver}

\subsubsection{Description of the experimental setup and computational domain}

The first set of validation cases were dedicated to the motion of floater without occurrence of the net structure in 2D. The purpose of the validation was to examine the performance of the motion solver for the floater, especially the different motion integration schemes. The experimental data we used were from Kristiansen (2010), where series of experiments were performed on wave interaction with a moored horizontal cylinder. The experiments were conducted in a narrow wave flume at the Department of Marine Technology, 
(b)
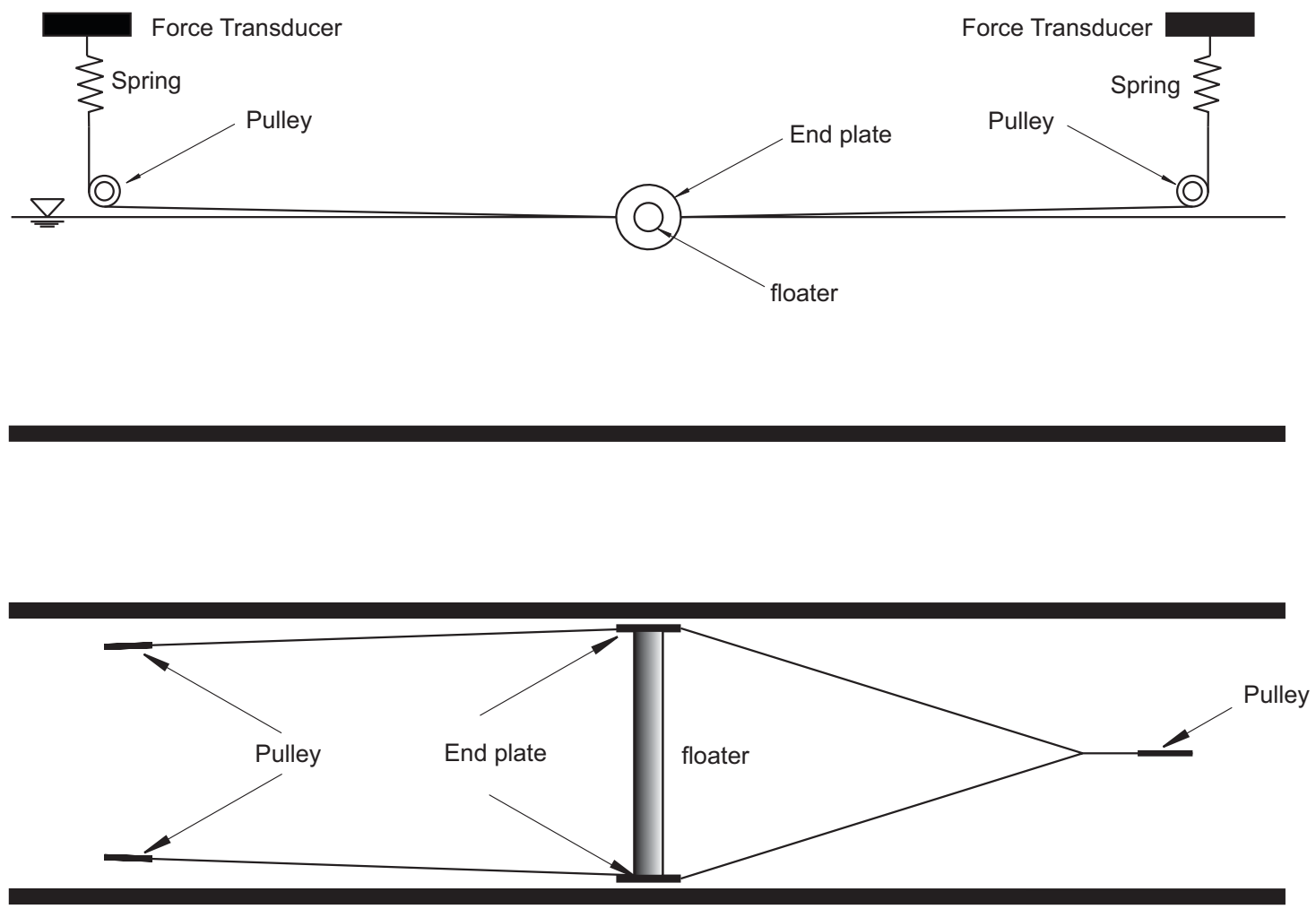

Figure 4: General setup for the experiments in Kristiansen $(2010)$ for wave interaction with moored floaters. (a) side view. (b) top view. The tank dimensions were $L \times B \times H=13.67 \times 0.6 \times 1.3 \mathrm{~m}$. A single-flap wavemaker was located at the far left end of the tank, and a parabolic beach was at the opposite end. The floater was positioned at the free surface equipped with end plates. On the wavemaker side, the floater was connected with two mooring lines which further connected to two pulleys. On the other side towards the beach, two mooring lines were first joined before connecting to the pulley.

Norwegian University of Science and Technology. A general sketch of the setup of the experiments is given in Fig. 4. The flume has a dimension of $13.67 \times 0.6 \times 1.3 \mathrm{~m}$, which was equipped with a single-flap wavemaker to generate waves. The water depth was set to be $1 \mathrm{~m}$. A circular horizontal cylinder was positioned at the free surface with diameter of $0.1 \mathrm{~m}$ and a length of $0.58 \mathrm{~m}$. At two ends of the cylinder, end-plates made from transparent plexi-glass were applied to reduce the 3D flow effects. This gave a $5 \mathrm{~mm}$ gap at each end between the end plate and the walls of the tank to avoid any contact. The cylinder was placed at a distance of $6.5 \mathrm{~m}$ from the hinge of the wave paddle and kept its position by four mooring lines. Each mooring line was pointing nearly horizontally. On the wave maker side, the two mooring lines were applied and connected to two pulleys. Meanwhile, on the other side towards the beach, two mooring lines were first joined to form a crowfoot before connecting to the pulley. Readers are referred to Kristiansen (2010) for further details of the experiment setup.

A 2D numerical wave tank was set up in order to reproduce the experiments. The simplified computational domain is shown in Fig. 5. The wave generation toolbox developed in Jacobsen et al. (2012) was applied to generate stream function waves. Two relaxation zones were applied at two ends of the tank to generate and absorb the waves. Since the end-plates were not modelled, an equivalent mass of $3.940 \mathrm{~kg} / \mathrm{m}$ was used in the numerical model for the circular cylinder with the end-plates. The mooring lines were not resolved but modelled as two linear springs, where the far end was pin-pointed at the coordinates of the contact point between the mooring line and the pulley. The other end of the mooring line was located at the model centre, and moving with the body. The equivalent mooring stiffness was set to $88.2 \mathrm{~N} / \mathrm{m}$. Five wave conditions were selected for validation purpose. The relevant wave parameters, Reynolds and KC numbers 


\section{atmosphere}

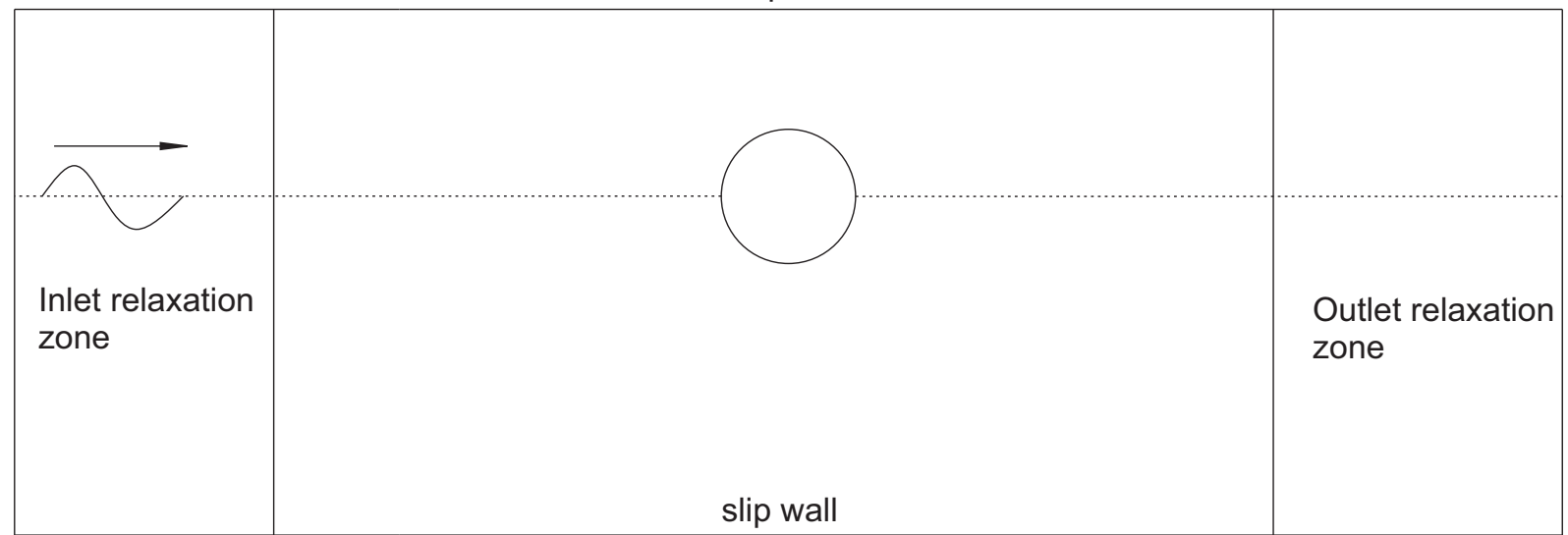

Figure 5: Sketch of the computational domain in the numerical model. Two relaxation zones were applied at two ends of the tank to generate and absorb the stream function waves. A slip wall boundary condition was assigned for the bottom of the numerical tank. At the top of the numerical tank, a commonly used atmosphere boundary condition was applied. The floater was located at the free surface. The mooring lines were not resolved, but modelled as linear springs.

Table 1: The parameters of the selected wave conditions for validation of the motion solver

\begin{tabular}{cccccc}
\hline Wave case no. & 1 & 2 & 3 & 4 & 5 \\
\hline Wave period, $T_{w}[\mathrm{~s}]$ & 0.497 & 0.544 & 0.601 & 0.761 & 0.878 \\
Wave height, $H_{w}[\mathrm{~m}]$ & 0.028 & 0.033 & 0.040 & 0.065 & 0.086 \\
Reynolds (Re) number [-] & $1.69 \times 10^{4}$ & $1.82 \times 10^{4}$ & $2.00 \times 10^{4}$ & $2.58 \times 10^{4}$ & $2.96 \times 10^{4}$ \\
Keulegan-Carpenter (KC) number [-] & 0.84 & 0.99 & 1.206 & 1.96 & 2.60 \\
\hline
\end{tabular}

are given in Table 1

\subsubsection{Convergence test}

Before we started the simulations, convergence analysis was first conducted. In the convergence analysis, free decay of the floater in heave direction was simulated. Initially, the floater had a displacement of $0.33 D$ from the equilibrium position. Then the floater was free to oscillate in heave direction. Three levels of mesh resolutions were applied, corresponding to 35000, 52000 and 76000 cells. The test results are presented in Fig. 6. It was found that the numerical solutions were monotonically convergent with increasing mesh resolution. Relatively small differences were observed between each of the solutions, demonstrating that the solution was insensitive to the mesh resolution. In the numerical simulations presented in Section 3.1.3. it was decided to apply the medium mesh resolution as the base mesh resolution. However, due to the difference of the waves that were generated in each case, the length of the relaxation zone was also different. In addition, the mesh resolution at the free surface was also refined properly for the cases with relatively small wave height. Essentially, the total number of mesh cells were determined by all these factors.

\subsubsection{Computational results}

The heave and sway motion of the floater under five wave conditions are given in Fig. 7- 11. Three different motion integration methods as described in Section 2.3 were tested for all the five wave conditions. The relevant results are reported in these figures. The results from leapfrog scheme were not reported, since we suffered from some stability issues for cases with steep wave conditions, which has been also mentioned in Section 2.3.

As presented in Table 1, the KC number is between 0-3, which indicates that the inertia effects are dominant. Viscous effects are secondary. However, when overtopping occurs, strong nonlinearity was found 


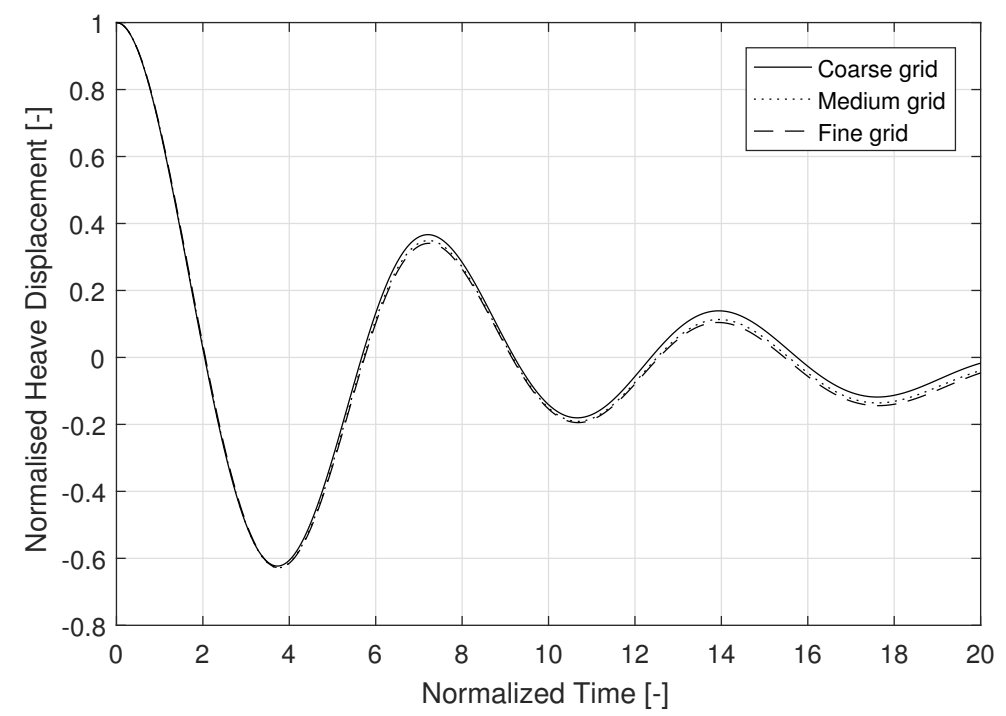

Figure 6: Heave motion as a function of time from the numerical free decay test. The heave displacement was nondimensionalized as $\eta_{3} / \eta_{3,0}$, where $\eta_{3,0}$ is the initial displacement of the floater in heave direction. The time was nondimensionalized as $t \sqrt{g / D}$.

on the free surface motions. Snapshots of the free surface wave and the floater at different time for wave case 5 are shown in Fig. 12, which clearly shows an overtopping process. In addition, during the overtopping process, the flow was locally separated from the boundary layer, where vorticity was induced. This creates a drag force, indicating that viscous effect plays a role. This has been explained in e.g. Kristiansen (2010) and Ong et al. (2017).

In terms of the performance of different integration methods, we found that no one performs consistently better in all the wave conditions than the others. The response amplitude operator (RAO) for heave and sway motion from the present model, the linear solution and the experiments in Kristiansen (2010) are given in Fig. 13. It should be mentioned that RAO could only partially reflect the performance of the different methods. The reason is that the amplitudes of nonlinear motions were obtained as the mean value of half of difference between the crest and the trough. Therefore, in principle the shift up and down of the numerical results produces the same RAO value.

It was observed that in relatively long waves, e.g. in wave case 5, the RAO value in heave motion is approaching one via linear solution. This means that the floater simply follows the evolution of the free surface waves. This is due to the fact that the ratio between the wave length and the floater diameter is rather large. Hereby the diffraction effect is negligible. However, in reality, overtopping created a quite irregular crest for the time series of heave motion, where dual peak may occur as shown in Fig. 11 . The RAO values from experimental data and numerical results also slightly deviate from one. Meanwhile, some scattering was found on the experimental data in this case. But the numerical results from all the integration methods are within the bound of the data. Regarding the performance of each methods, we found that in such long waves, especially Crank-Nicolson scheme and the Newmark scheme have very similar performance. Adams-Bashforth-Moulton scheme also produced similar results except in wave case 5 .

In shorter wave conditions, e.g. in wave case 1 and 2 , the numerical results from different integration methods are distinguishable. For wave case 2, as described in Kristiansen (2010) instability was observed in the experiments, where the sway motion was increasing until the motion became significantly large and violent that contact between the model and the side walls occurred. Hereby the RAO value in sway mode in wave condition 2 is not plotted in Fig. 13 . But in Fig. 8, by direct comparison of the time series of the sway motion, it was noticed that the same phenomenon was also observed from the numerical results. However, the sway motion was amplified more significantly than in the experiments. Among all the integration methods, Newmark integration produced the most reasonable estimation. For wave case 1, the steady state condition was reached in both experiments and numerical simulations. In this case, Adams-Bashforth-Moulton scheme 

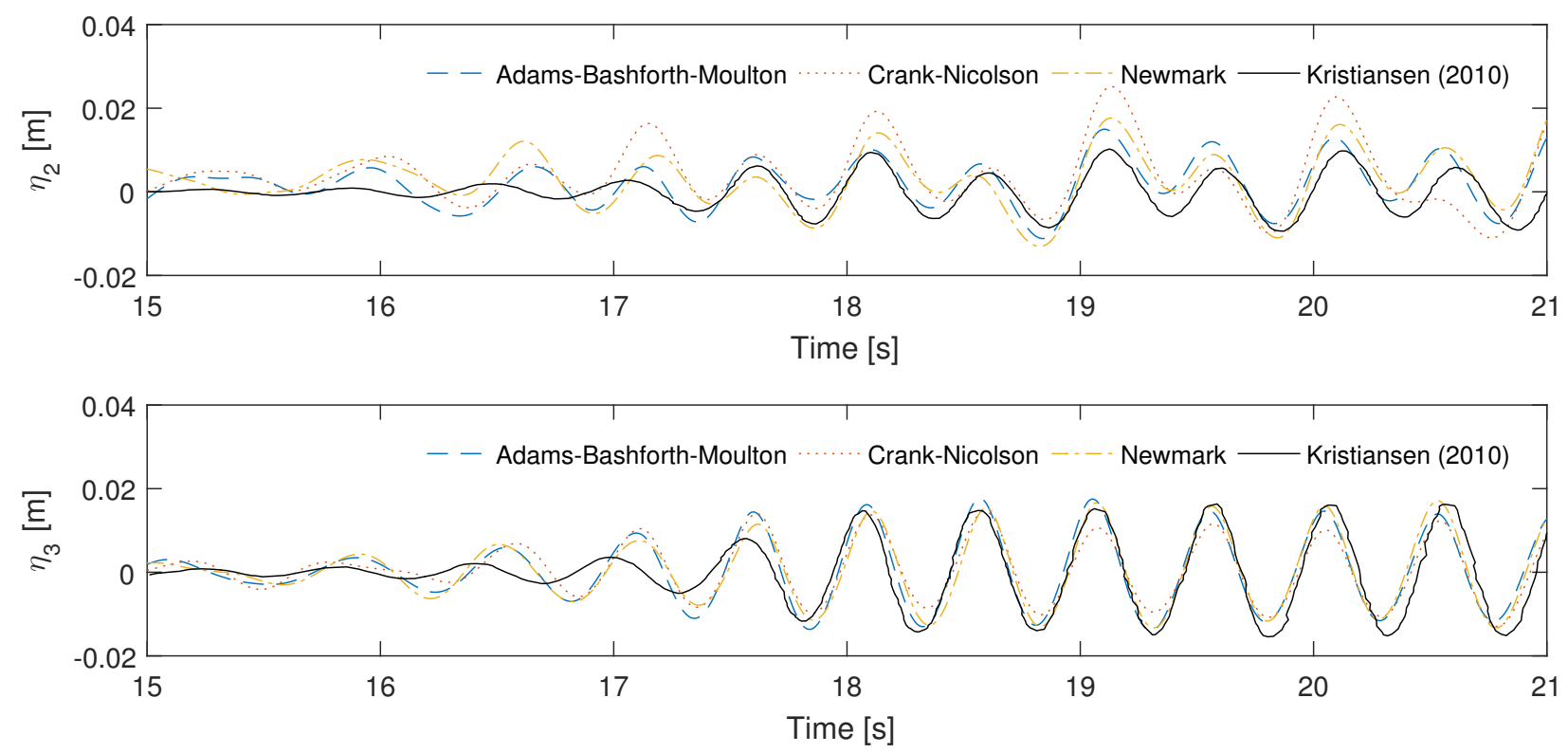

Figure 7: Comparison of sway $\left(\eta_{2}\right)$ and heave $\left(\eta_{3}\right)$ motion of the floater from the present numerical model and the experiments in Kristiansen (2010) for wave case 1 with $H_{w}=0.028 \mathrm{~m}$ and $T_{w}=0.497 \mathrm{~s}$.

gave a better prediction. In both cases, Crank-Nicolson scheme gave the worst predictions. In the following part in Section 3.3. for simulating the floater-net system in waves and combined current and waves, the Adams-Bashforth-Moulton scheme was chosen as the integration method for floater motion equation.

\subsection{Validation of the net solver}

\subsubsection{Description of the experimental setup and computational domain}

The hydrodynamic and structural solver for the net were validated against a set of experiments in Bardestani and Faltinsen (2013) for plane flexible net panels in steady current flow. The experiments were performed in the same wave flume as in Kristiansen (2010). In the experiments, three different net panels were applied. The nets were made from Raschel material with square meshes. The unstretched length and width of the net panels were $0.76 \mathrm{~m}$ and $0.51 \mathrm{~m}$, respectively. The solidity ratio and the diameter of the twines are given in Table 2. Furthermore, three different sinkers were attached in the bottom of the net panel with the mass $W_{s}=1.2,1.4$, and $1.6 \mathrm{~kg}$. The diameter and the length of the sinker were reported as $5 \mathrm{~cm}$ and $55 \mathrm{~cm}$. No floater was involved in this set of experiments. Instead, the net was top fixed and deformed freely under steady current.

According to the experiments, we set up a 2D numerical model. A snapshot of the computational domain and computational mesh is given in Fig. 14. The net was represented by a sheet of moving porous media, where the thickness is $50 \mathrm{~mm}$. The porous resistance coefficients were calculated based on Eq. 16- 18), and the results are reported in Table 2. The bottom wall effect was neglected, where the wall was treated as a slip wall. This is due to the fact that the boundary layer developed at the bottom has minor effect on the net. The water depth in the numerical model was the same as in the experiments. However, the length was shortened to save the computational time. The mesh near the net region was refined, in order to better capture the geometry of the net.

In addition, it was noticed that in this set of experiments, the diameter of the sinker was significantly larger than the twine diameter. This indicated that the bottom effect might be important. Since the sinker was not modelled, only the forces were added to the structural model. Therefore, selection of the drag force coefficient for the sinker is important. In reality, the net was connected to the sinker, which might alternate the pressure distribution and the separation point. Bardestani and Faltinsen (2013) mentioned that in such a condition, where the cylinder was connected with a tripping wire, considerable increase of the 

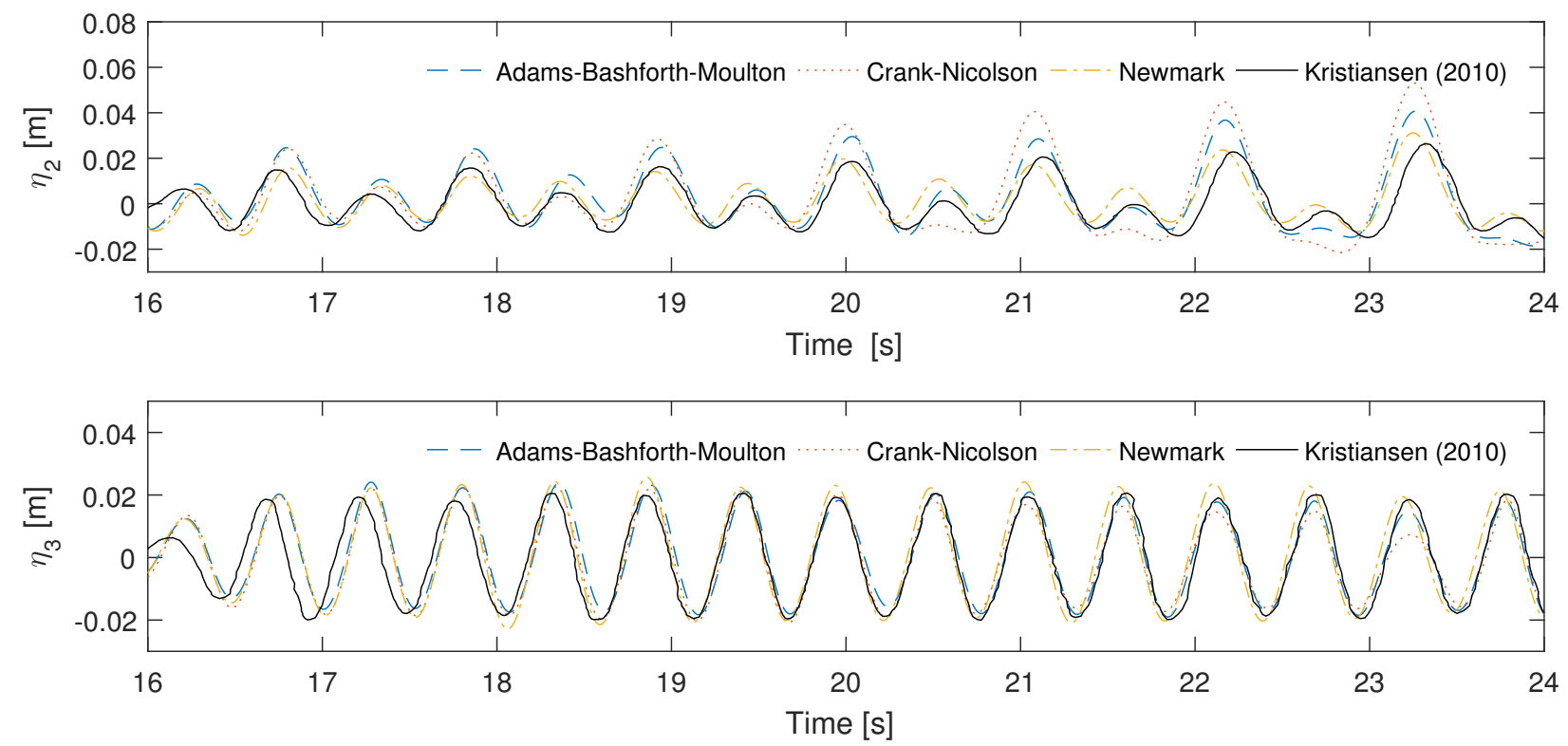

Figure 8: Same as in Fig. 7 but for wave case 2 with $H_{w}=0.033 \mathrm{~m}$ and $T_{w}=0.544 \mathrm{~s}$.
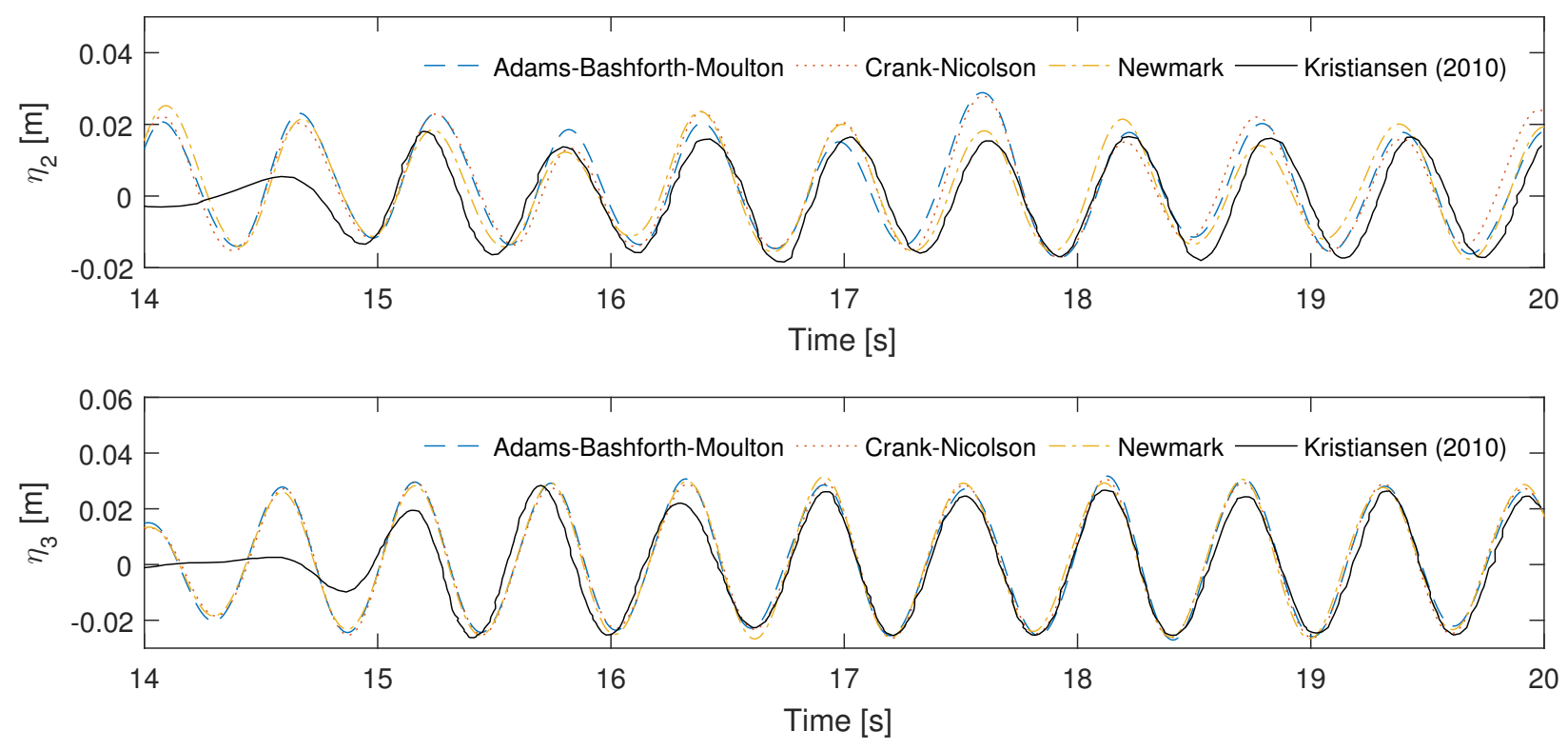

Figure 9: Same as in Fig. 7] but for wave case 3 with $H_{w}=0.040 \mathrm{~m}$ and $T_{w}=0.601 \mathrm{~s}$.

Table 2: The parameters and calculated porous resistance coefficients for the three nets

\begin{tabular}{cccccc}
\hline Net case no. & $d_{\text {twine }}[\mathrm{mm}]$ & $S_{n}[-]$ & $C_{1}[-]$ & $C_{2}[-]$ & $C_{3}[-]$ \\
\hline 1 & 2.5 & 0.16 & 6.7183 & 2.2409 & 2.2815 \\
2 & 2.5 & 0.19 & 8.0900 & 2.5693 & 2.6108 \\
3 & 1.8 & 0.23 & 10.5974 & 3.1604 & 3.1894 \\
\hline
\end{tabular}



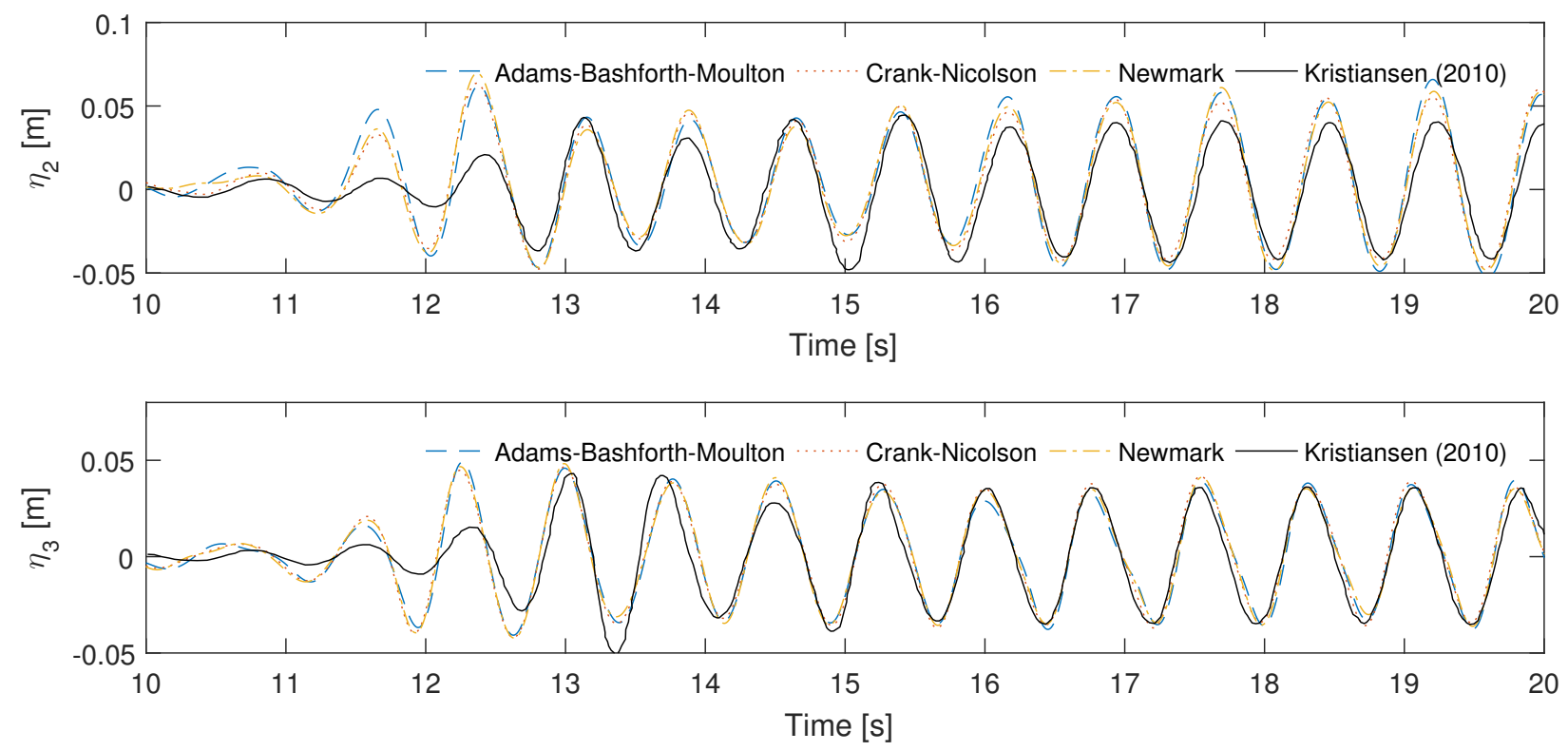

Figure 10: Same as in Fig. 7) but for wave case 4 with $H_{w}=0.065 \mathrm{~m}$ and $T_{w}=0.761 \mathrm{~s}$.
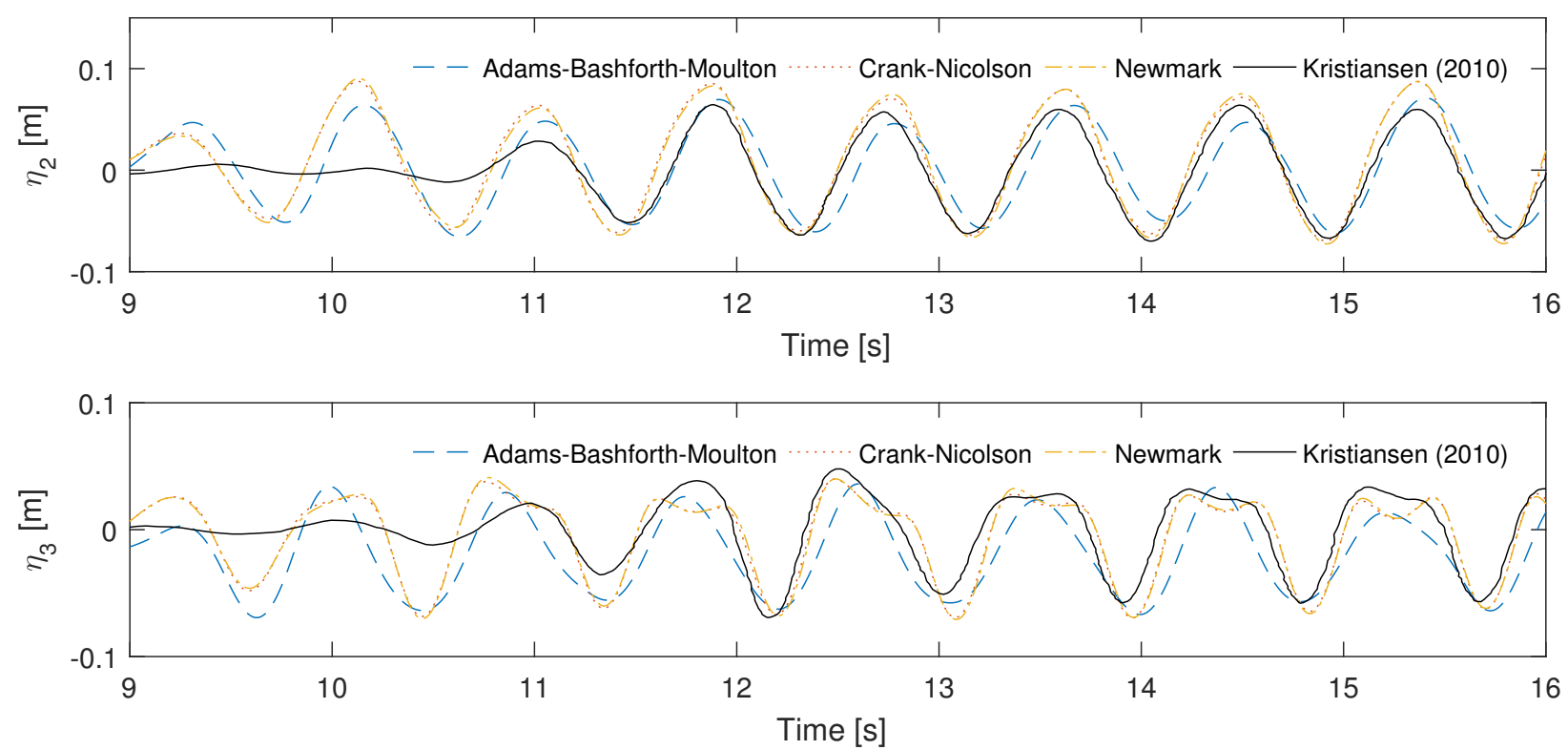

Figure 11: Same as in Fig. 7. but for wave case 5 with $H_{w}=0.086 \mathrm{~m}$ and $T_{w}=0.878 \mathrm{~s}$. 


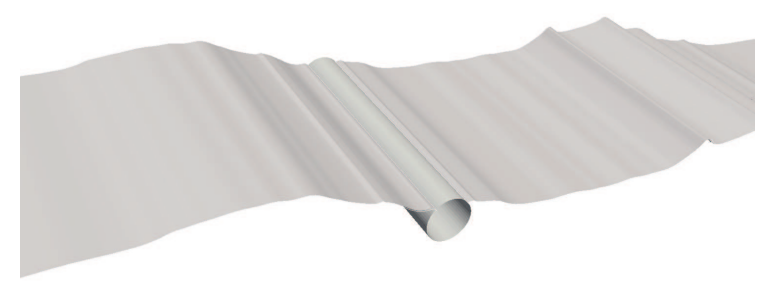

Time: $19.0 \mathrm{~s}$
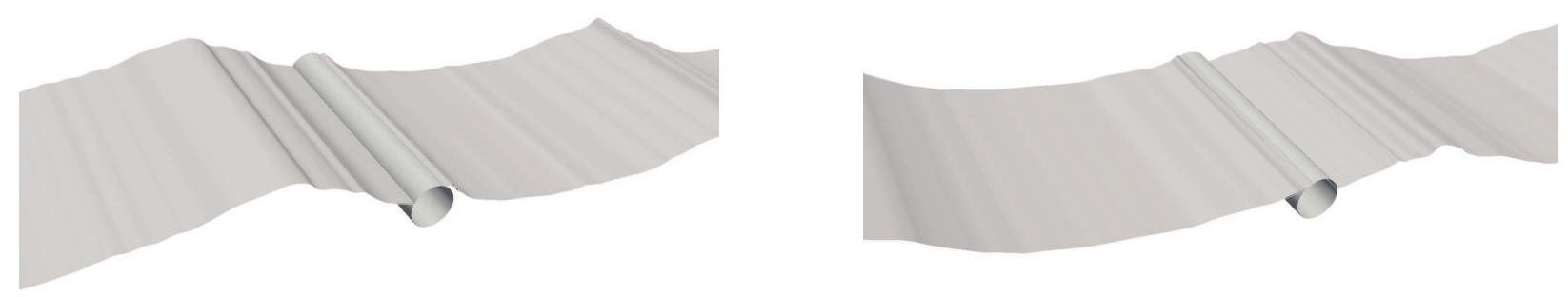

Figure 12: Snapshot of the free surface wave and the floater at different time for wave case 5.
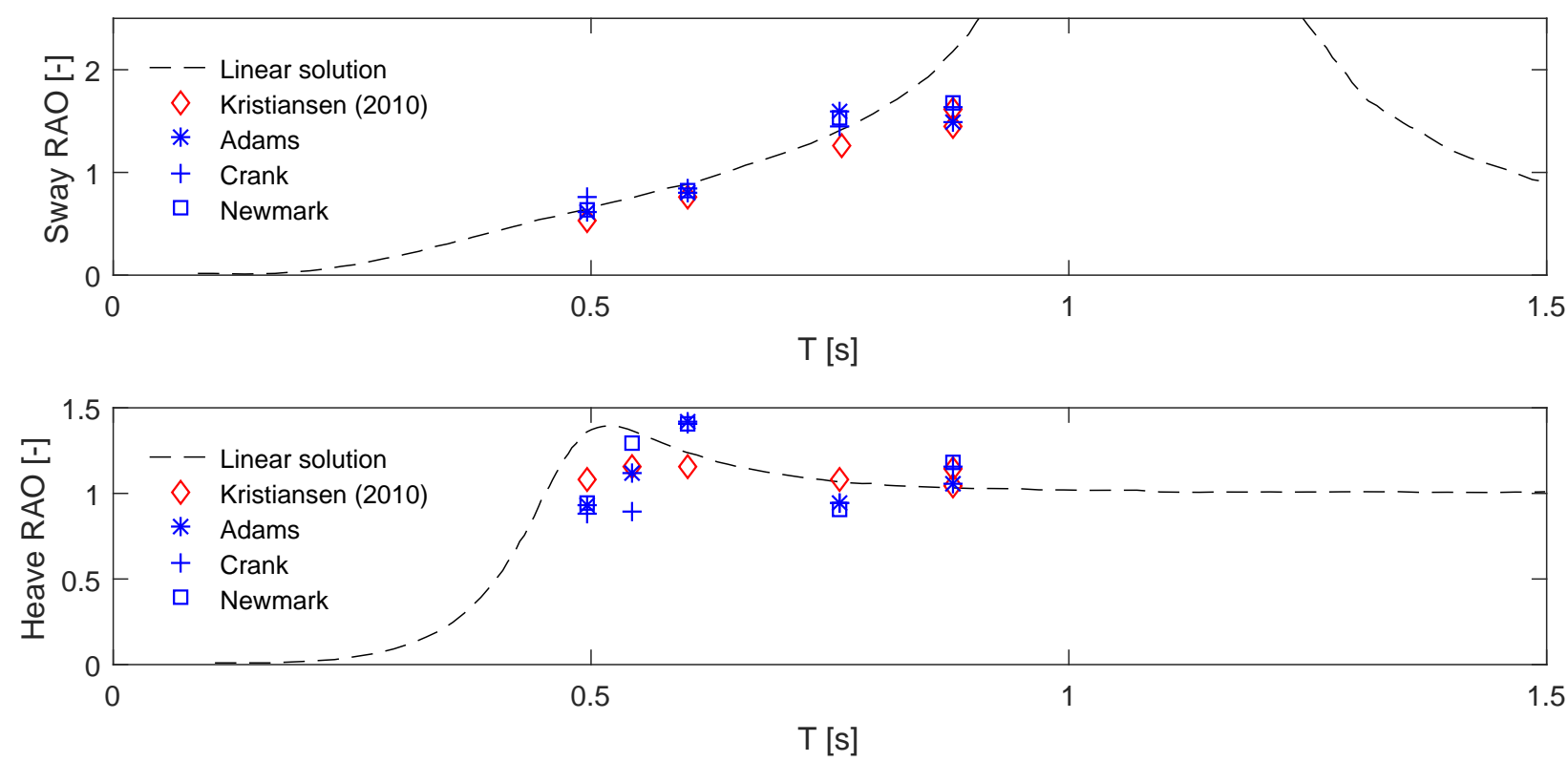

Figure 13: Comparison of the response amplitude operator (RAO) for heave and sway motion between linear solution, experimental data from Kristiansen (2010) and the present model with different motion integration methods. 
Figure 14: The computational domain and computational mesh for reproduction of experiments in Bardestani and Faltinsen (2013) for plane net panel in steady current. The origin of the coordinate system was located at the center of the top layer of the net panel. The mesh in the vicinity of the net panel was refined.

drag coefficient may be expected. Some relevant results have been reported in James and Truong (1972), according to which we used a drag force coefficient of 1.8 here for all the cases presented in Sec 3.2.3

\subsubsection{Sensitivity analysis on the drag force coefficient of the sinker}

As mentioned above, due to the complex flow condition, a large uncertainty was expected with regard to the selection of the drag force coefficient for the sinker. Following the suggestions given by our reviewers, we conducted uncertainty analysis for the drag force coefficient. The nominal drag force coefficient was chosen as 1.8, and a $30 \%$ uncertainty was assigned for this coefficient. This means that the drag force varies between 1.26 and 2.34. The cases that were selected for the uncertainty analysis are with the solidity ratio $S_{n}=0.16$, the sinker mass $W_{s}=1.2 \mathrm{~kg}$, and the incoming velocities $U_{c}=0.1,0.2,0.3 \mathrm{~m} / \mathrm{s}$.

The results from the numerical uncertainty analysis are presented in Fig. 15. The variation of the tension force $\Delta F$ was computed as

$$
\Delta T_{n}=\frac{1}{2} \sum_{i=1}^{2}\left|T_{n, i}-T_{n, 0}\right|
$$

where $T_{n, 0}$ is the nominal tension force. From Fig. 15, it is seen that for the case with $U_{c}=0.1 \mathrm{~m} / \mathrm{s}$, the drag force coefficient for the sinker has rather insignificant effects on the final tension force. The difference is $2 \%$ with a variation of $30 \%$ for the drag force coefficient. However, with the increase of the incoming velocities, the selection of the drag force coefficient starts to play a role. For the case with $U_{c}=0.3 \mathrm{~m} / \mathrm{s}$, essentially the variation of the tension force reaches $14.5 \%$. Therefore, care should be taken on selection of drag force coefficients in such cases.

\subsubsection{Computational results}

Examples of the net deformation are given in Fig. 16 for the cases with $S_{n}=0.19$, the current velocity $U_{c}=0.2 \mathrm{~m} / \mathrm{s}$, and the sinker mass $W_{s}=1.2,1.4$ and $1.6 \mathrm{~kg}$. The experimental results and the results from the screen type force model are given in Bardestani and Faltinsen (2013). Readers are referred to this paper for 

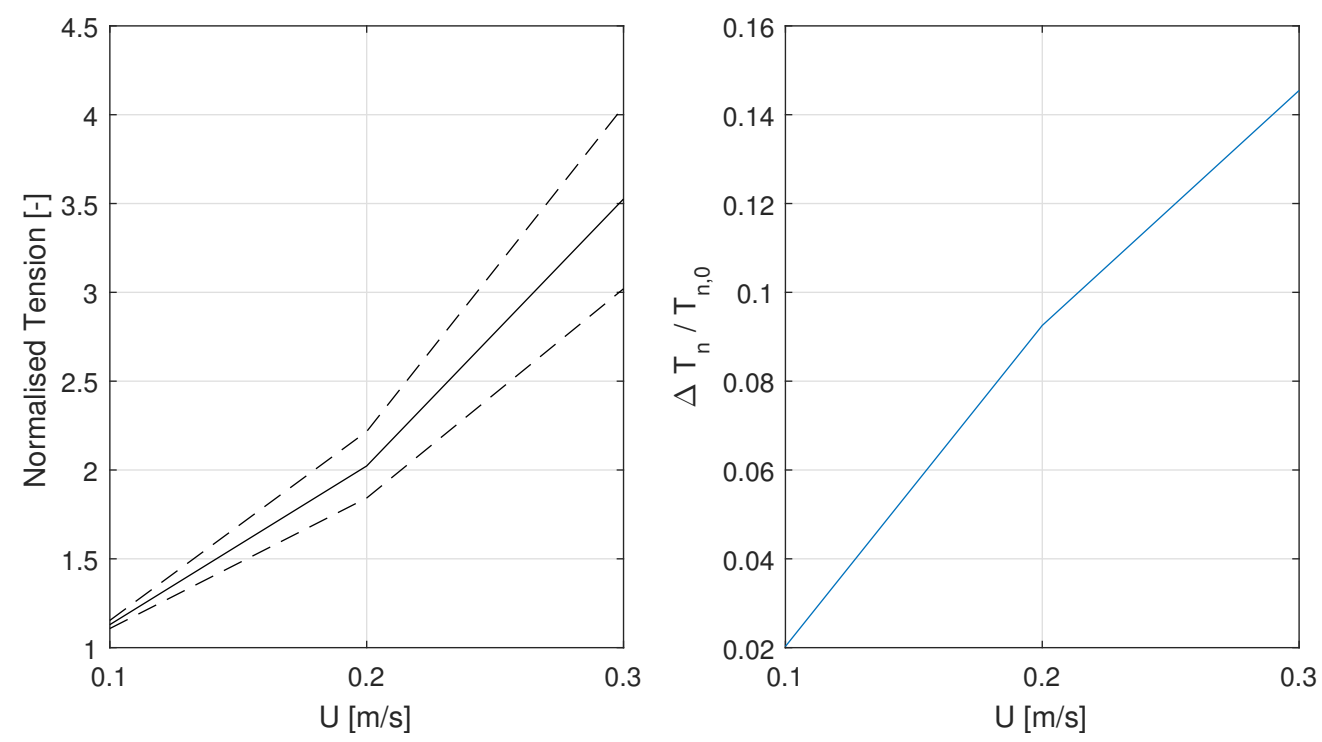

Figure 15: Results from numerical uncertainty analysis for the drag force coefficient of the sinker. The left figure presents the upper and lower bound of the normalized tension force due to the variation of the drag force coefficient for the cases with $S_{n}=0.16$ and $W_{s}=1.2 \mathrm{~kg}$. The right figure shows the relative error normalized by the nominal value for the same cases.

the photograph of the net deformation in the experiments. In general, by qualitative comparison between the present model, the screen type force model and the model from the experiments, we concluded that the results from our numerical model and the screen type force model agree well with the experiments. In Bardestani and Faltinsen (2013), the figures from the experiments were not given in a coordinate system. Therefore, quantitative comparison is not possible. But it was observed that our numerical model consistently predicted a slightly larger deformation than the screen type force model. As mentioned in Bardestani and Faltinsen (2013), due to use of too few elements, the screen type force model slightly underestimated the deformation. So, in principle, our numerical model should produce a slightly better result in this case.

Comparison of the tension force is given in Fig. 17 between our numerical model and the experimental data in Bardestani and Faltinsen (2013). In general, a very good agreement was found. The largest error occurs for the case with $W_{s}=1.2 \mathrm{~kg}, S_{n}=0.16$ and $U_{c}=0.3 \mathrm{~m} / \mathrm{s}$, where the error reaches up to $20 \%$. For the rest cases, the errors are all below or around $10 \%$.

\subsection{Validation of the floater-net system}

The final validation cases are related to the whole floater-net system under various wave conditions and combined current and wave conditions. The experiments have also been conducted in Bardestani and Faltinsen (2013) in the same current-wave flume as the experiments in Section 3.1 and Section 3.2. In this set of experiments, the floater is the same as the one used in Kristiansen $(2010)$, while the mass of the sinker is $1.6 \mathrm{~kg}$. In the experiments under regular waves, the solidity ratio of the net was 0.23. But under combined current and wave condition, the net panel with $S_{n}=0.16$ was used. Both of them have been tested in Section 3.2. The net was connected to the floater using strips.

Again, we set up our numerical model based on the the experiments, which was a combination of the setup of the numerical model in Section 3.1 and 3.2 A body-fitted mesh was applied in the computational domain, where the floater was described as a boundary patch in the flow domain. The net was modeled as a sheet of porous media, which was attached on the floater. The numerical wave tank used in this set of simulations is the same as used in Section 3.1. while the parameters for the net and sinker system are the same as in Section 3.2. A snapshot on the initial configuration of the numerical model is given in Fig. 18 . 

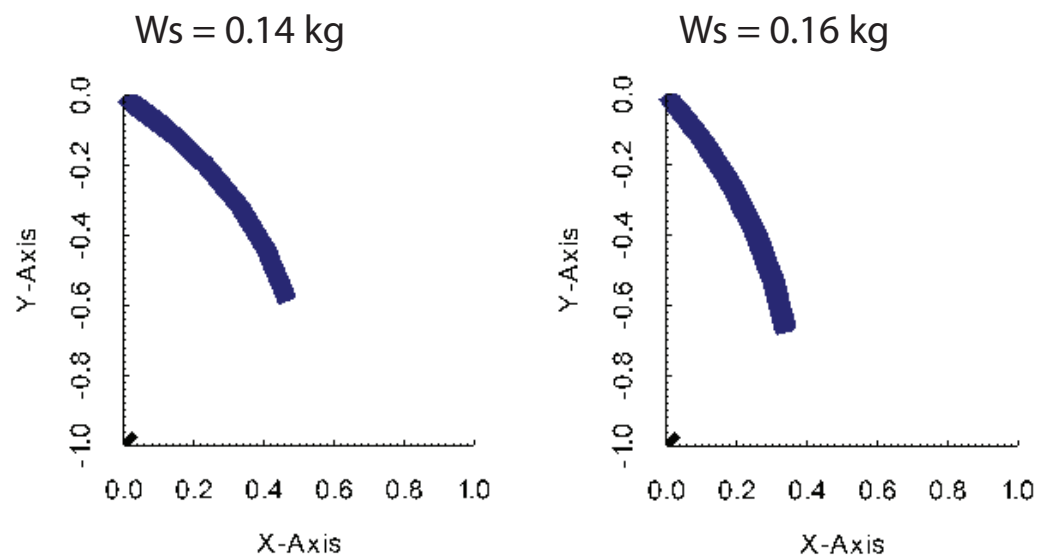

Figure 16: The deformation of the net panel with $S_{n}=0.19, W_{s}=0.12,0.14$ and $0.16 \mathrm{~kg}$ under the incoming velocity $U_{c}=0.2$ $\mathrm{m} / \mathrm{s}$.

Table 3: The parameters of the selected wave conditions for validation of the floater-net system in regular waves

\begin{tabular}{cccccc}
\hline Wave case no. & 1 & 2 & 3 & 4 & 5 \\
\hline Wave period, $T_{w}[\mathrm{~s}]$ & 0.497 & 0.601 & 0.672 & 0.878 & 1.038 \\
Wave height, $H_{w}[\mathrm{~m}]$ & 0.028 & 0.040 & 0.050 & 0.086 & 0.120 \\
\hline
\end{tabular}

\subsubsection{Snap load of the net under regular wave condition}

For the cases with floater-net system under regular wave conditions, the tension force of the net was measured in the experiments. Five force transducers were installed at the top layer of the net, and the average values were reported. A very large snap load was observed for most of the cases. The form of the snap load was closely related to the relative motion between the floater and the sinker, and the characteristics of the net material property. Physically, at some specific time in a wave period, due to the relative motion between the floater and the sinker, the distance between them was smaller than at hydrostatic condition. Due to the negligible compression stiffness, the net got slack at this time. After that, the floater was moving upwards while the sinker was accelerating downwards. When the net got taut again, very large snap load occurred.

In the present work we numerically reproduced the snap load of the system under five wave conditions, as shown in Table 3. The time series of the snap load under these wave conditions are given in Fig. 19 Several comments are given for the numerical results: (1) In all the cases, at the beginning of the simulation before the waves reaches the floater, (approximately from $0-4 \mathrm{~s}$, depending on the propagating speed of the incoming wave), there still exist periodic cycles for the tension force. The reason is that initially the system was not exactly in equilibrium state. Small amplitude free decay motion was observed. Therefore, the oscillation period at this stage should be related to the natural period of this floater-net system where the wet weight of the sinker was added. This could also be demonstrated by the fact that in all the cases, the oscillation periods at this stage are identical, irrespective of the incoming wave conditions. (2) There exists two or several peaks within one wave period. This indicates that after the net became taut from slack status for the first time, strong internal force was generated, which is the reason for occurrence of the snap load. This internal force caused the net to get slack again and later produced the second peak. However, in principle, the second peak should be smaller than the first peak, since the energy was dissipated between occurrence of two peaks. It was noticed that for some cases, e.g. the case with $T_{w}=0.497 \mathrm{~s}$ and $H_{w}=0.028$ $\mathrm{m}$, the second peak has rather high value. This might be due to neglecting modelling of the sinker, where the damping effects of the sinker was not properly considered in the numerical model. (3) The period between two largest peaks is the wave period.

Accurate prediction of this type of snap load is not straightforward. It requires that both the motion of 

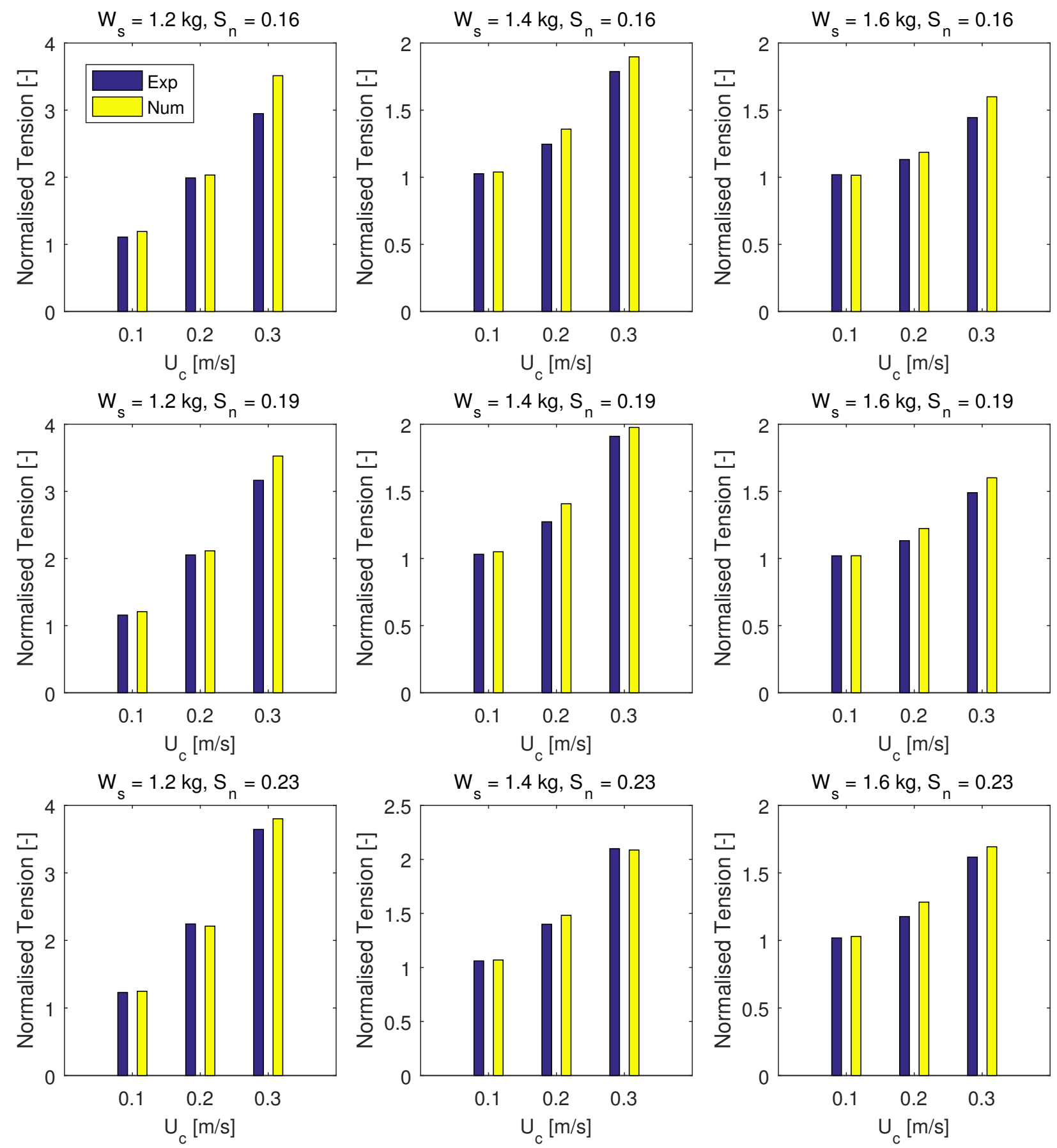

Figure 17: Comparison of the tension forces on the top layer twines of the net panel between the experimental data in Bardestani and Faltinsen (2013) and the present numerical model. The tension force was normalized by the initial tension. 


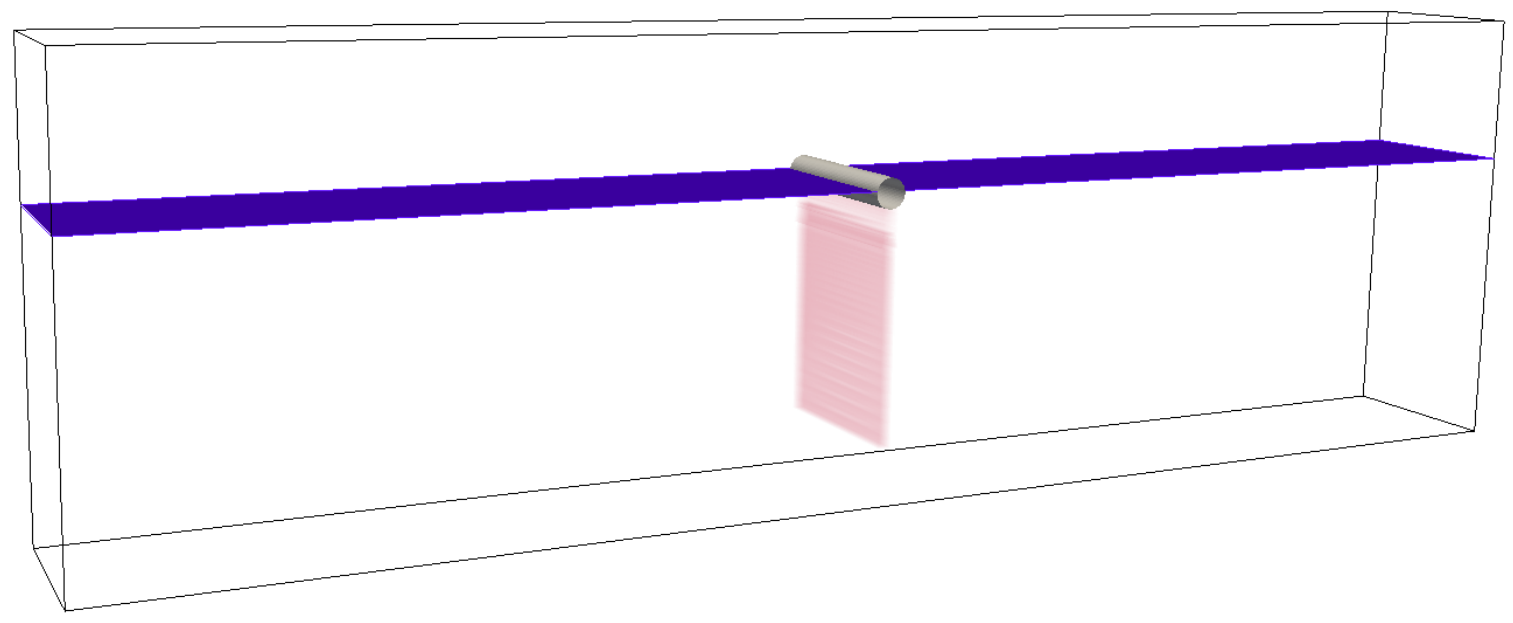

Figure 18: The initial configuration of the numerical model for simulation of floater-net system. The floater was placed at the still free surface. The porous zone which represented the net panel was attached on the floater.

the floater and the sinker should be predicted accurately. Meanwhile, the structural model of the net should reflect the property of the net material precisely. Comparison of the average amplitude of the snap load is given in Fig. 20. It is seen that the snap load is several times larger than the load at hydrostatic condition, which is about $5 \mathrm{~N}$. Some of the scattering of the experimental data was also observed under the same wave condition.

In Fig. 20 we noted some deviations between the results from the present numerical model and the experimental data. The possible reasons for the deviations are given as follows. In the numerical model, the sinker geometry was not resolved, and the hydrodynamic force on the sinker was neglected. A rough estimation is given here on the magnitude of the hydrodynamic force on the sinker. The force was estimated based on Eq. (3) and Eq. (4). It was assumed that the sinker and the floater were connected rigidly, i.e. the net behaves like a rigid bar. Then the floater and the sinker have the same displacement. Furthermore, it was assumed that the floater follows the wave motion, i.e. the RAO is one. Therefore, the sinker motion was the same with the water particle in the free surface. Based on linear wave theory, it was expressed as:

$$
y_{s}=\frac{H_{w}}{2} \cos \omega_{w} t
$$

The sinker velocity then is the same as the particle velocity at the free surface:

$$
\begin{gathered}
U_{s}=\frac{g k_{w} H_{w}}{4 \pi f_{w}} \cos \omega_{w} t \\
V_{s}=\frac{g k_{w} H_{w}}{4 \pi f_{w}} \sin \omega_{w} t
\end{gathered}
$$

while $U_{w}$ and $V_{w}$ are the horizontal and vertical undisturbed wave velocity at that depth:

$$
\begin{aligned}
U_{w} & =\frac{g k_{w} H_{w}}{4 \pi f_{w}} \frac{\cosh \left(k_{w}\left(y_{s}+h\right)\right)}{\cosh \left(k_{w} h\right)} \cos \omega_{w} t \\
V_{w} & =\frac{g k_{w} H_{w}}{4 \pi f_{w}} \frac{\sinh \left(k_{w}\left(y_{s}+h\right)\right)}{\sinh \left(k_{w} h\right)} \sin \omega_{w} t
\end{aligned}
$$

Fig. 21 gives the estimated hydrodynamic forces for the case with $H_{w}=0.086 \mathrm{~m}, T_{w}=0.878 \mathrm{~s}$, which gives a peak value of $2.93 \mathrm{~N}$. Meanwhile, the snap load from the experiments for this case produces a mean 
value of $26.5 \mathrm{~N}$, as shown in Fig. 20. The peak of hydrodynamic force takes $11 \%$ of the total snap load. Although we should emphasize that this is case dependent, from this study, a rough indication can be given on the order of magnitude of the error that is introduced due to this simplification.

In addition, the constitutive relationship of the net material used in the present model is another error source. As mentioned above in Section 2.5, this relation was obtained from the experiments for one specific net, where the coefficients were determined by least-square fitting. However, since the nets used in Lader and Fredheim (2006) and Bardestani and Faltinsen (2013) are different, this set of coefficients used in the numerical model may not precisely reflect the material property of the net used in Bardestani and Faltinsen (2013). Furthermore, the oscillations of the floater-net system at the beginning of the simulations induced some wave radiations. The radiated waves might pollute the incoming waves, which introduced errors. However, the amplitude of the radiated waves was rather small, which should not have significant impact on the final results, especially in rather high wave conditions, e.g. wave case 4 and 5 .

\subsubsection{Mooring line forces under combined current and wave condition}

Another validation case was carried out under combined current and wave condition, where the wave height $H_{w}=0.065 \mathrm{~m}$, wave period $T_{w}=0.761 \mathrm{~s}$ and the current velocity $U_{c}=0.2 \mathrm{~m} / \mathrm{s}$. In the experiments, the combined current and wave condition was generated by first towing the net panel and the floater towards the wave maker side until a steady state condition was reached. Then the incident regular waves were generated, where the net panel already had an offset before the waves propagated through it.

In the numerical model, exact reproduction of the above experimental setup was not possible. The reason is that with a body fitted mesh grid, it was not possible to move the floater towards the wave maker side for a long time to reach the steady state condition. Therefore, we chose to directly generate the combined current and waves from the wave maker side, based on the high order stream function wave theory in Fenton (1988) with a drift velocity. However, one should note that the wave frequency was changed to the encounter frequency $\omega_{e}$ as shown below:

$$
\omega_{e}=\omega_{w}-\frac{\omega_{w}^{2} U_{c}}{g}
$$

A snapshot of the floater-net system and the free surface wave is given in Fig. 22, It was observed that within one wave period, the net deformation was largely influenced by the current velocity. On the other hand, the wave has minor influences. This was determined by the magnitude of the wave and current velocities at the position of the net. We could estimate the particle velocity induced by the waves using linear wave theory. Fig. 23 compares the magnitude of the current and amplitude of the wave velocity at various depth from free surface to the bottom of the net panel. It was noticed that with the increase of the depth, the wave velocity is decreasing significantly. From approximately $y=-0.05 \mathrm{~m}$, the current velocity starts to exceed the wave velocity. At the bottom of the net panel where $y=-0.81 \mathrm{~m}$, the maximum horizontal velocity of the water particle was estimated as $0.0013 \mathrm{~m} / \mathrm{s}$, which was around $0.65 \%$ of the current velocity. Therefore, the current completely dominated the deformation of the net. However, both the current and the wave played important roles on the motion of the floater.

We compared the mooring line forces between the experimental data in Bardestani and Faltinsen (2013) and the present model, which is given in Fig. 24. It was observed that the mean value is not zero due to co-existence of the current. Actually, the mean mooring line force is approximately the same with the drag force on the panel under current only condition. The oscillation part is then due to the wave that travels on the current. The experiment and the simulation gave approximately the same mean force on the mooring lines, which are $3.50 \mathrm{~N}$ and $3.06 \mathrm{~N}$, respectively. But the oscillation amplitude was underestimated by the present model, and some irregular characteristics were also observed from the numerical result.

\section{Summary and conclusions}

In the present work, a novel numerical model was developed to simulate the responses of a floater-net system in current and waves. It was an extension of our previous work in Chen and Christensen (2016) and Chen and Christensen (2017). The main contribution of the paper is that the motion of the floater was 

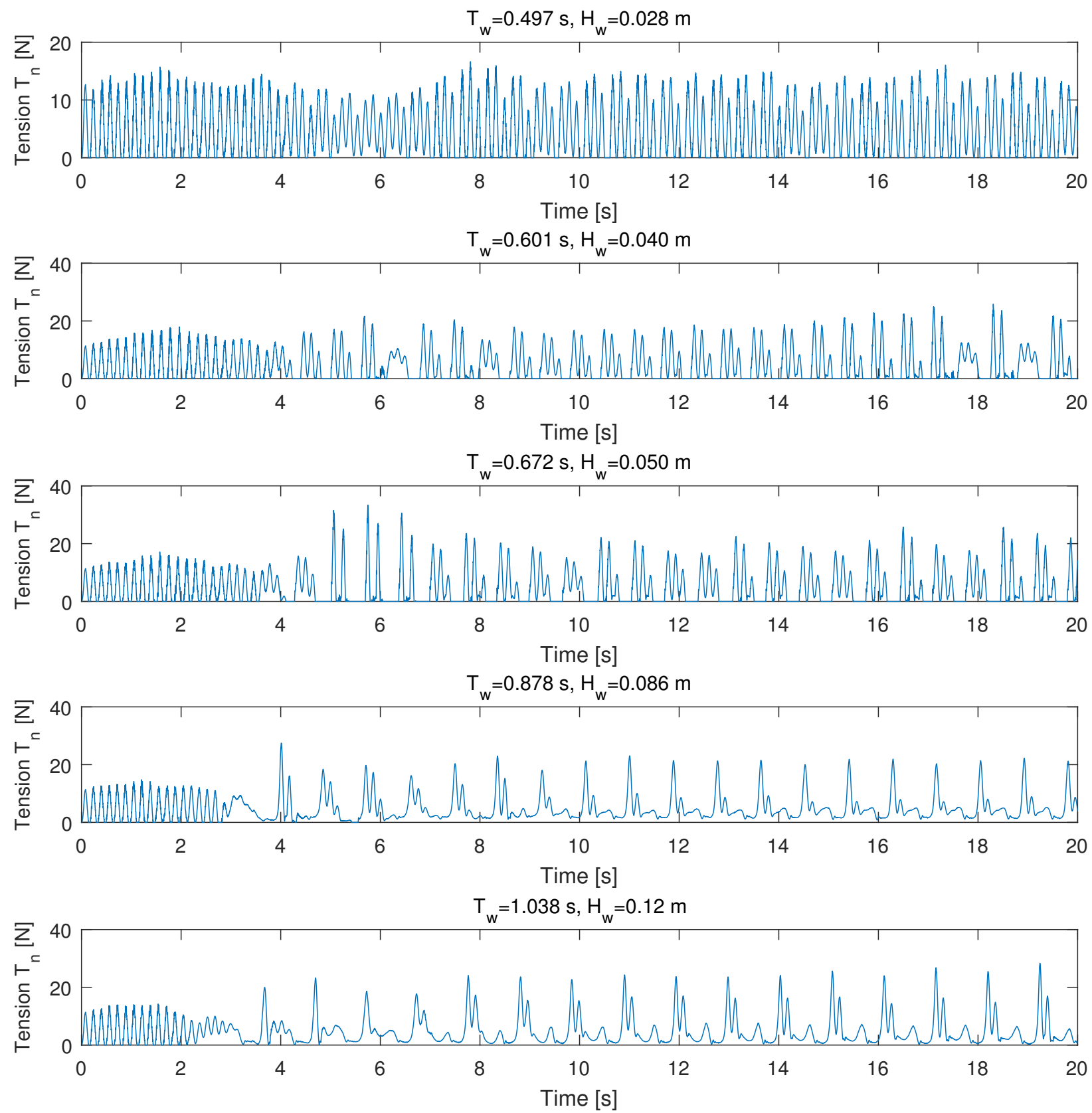

Figure 19: Time series of the tension force from the present numerical model under different wave conditions. 
Figure 20: Comparison of the amplitude of the snap load under different wave conditions between the present model and the experiments in Bardestani and Faltinsen (2013). The wave condition is listed in Table 3

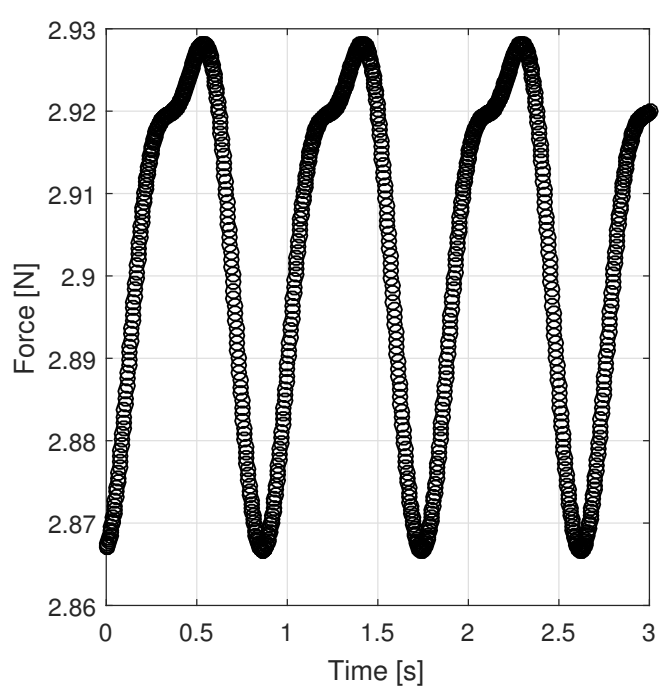

Figure 21: The estimated hydrodynamic force on the sinker for the case with $H_{w}=0.086 \mathrm{~m}, T_{w}=0.878 \mathrm{~s}$. The floater and the sinker were assumed to be connected rigidly. Furthermore, it was assumed that both of them follows the wave motion at the free surface. The hydrodynamic force was calculated based on Eq. (3) and Eq. 44. 
Figure 22: A snapshot on the free surface and the response of floater-net system at different time in combined current and wave condition. The wave period $T_{w}=0.761 \mathrm{~s}$, wave height $H_{w}=0.065 \mathrm{~m}$ and the current velocity $U_{c}=0.2 \mathrm{~m} / \mathrm{s}$.

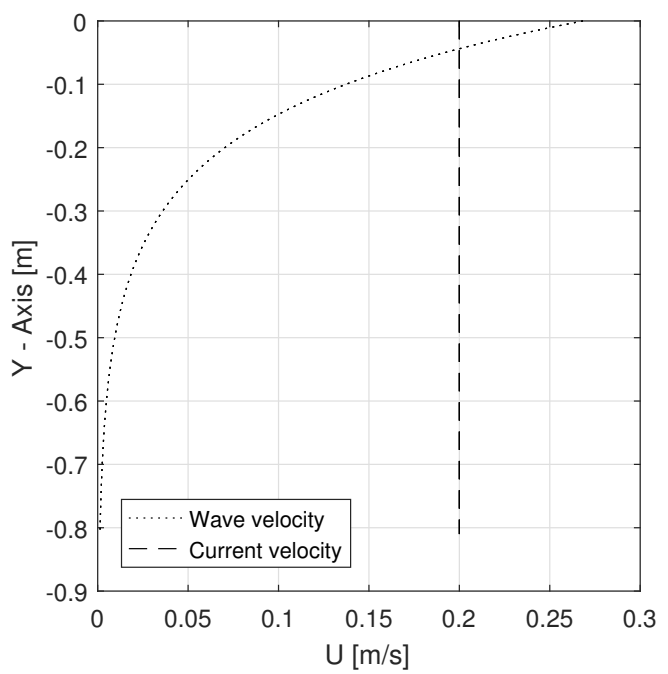

Figure 23: Comparison of the current and wave velocity of the water particle at various depth. The wave velocity was calculated via linear wave theory. The depth varies from free surface $(y=0 \mathrm{~m})$ to the bottom of the net panel $(y=-0.81 \mathrm{~m})$. 


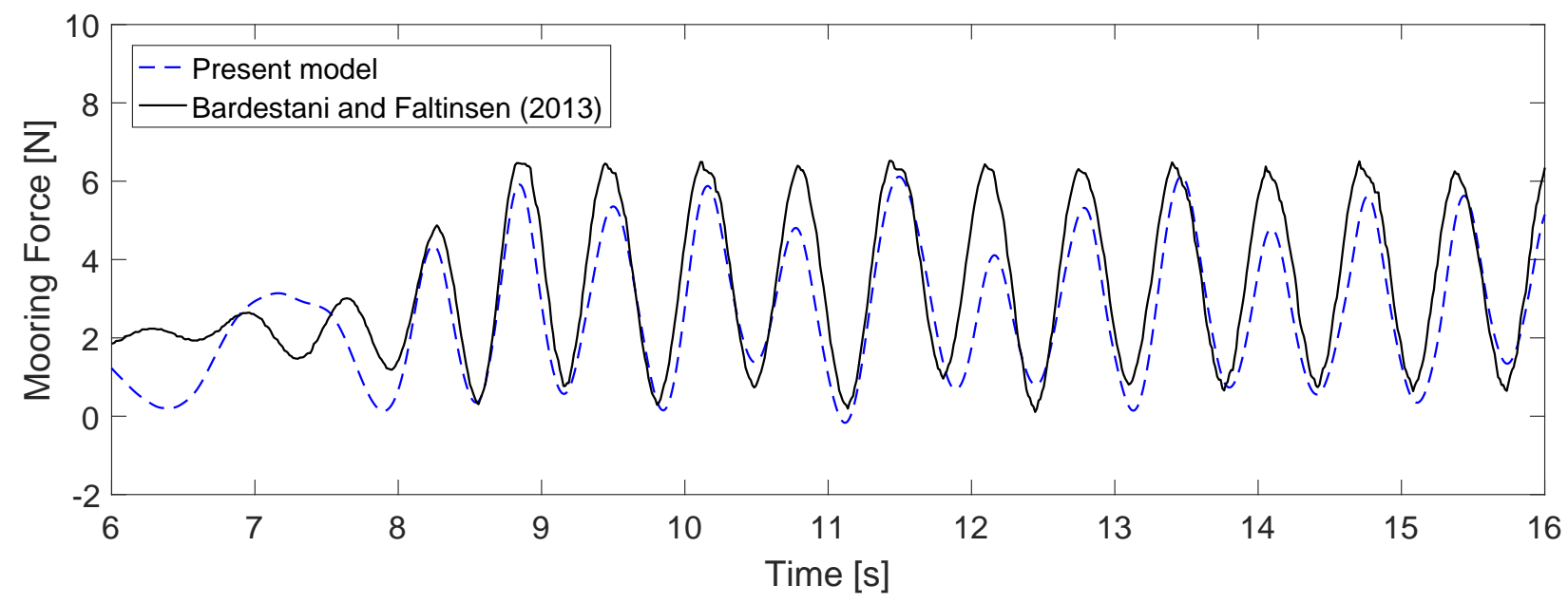

Figure 24: Comparison of the mooring line forces between the present numerical model and the experimental data from Bardestani and Faltinsen (2013) under combined current and wave condition. The current velocity $U_{c}=0.2 \mathrm{~m} / \mathrm{s}$, the solidity ratio $S_{n}=0.16$, the wave height $H_{w}=0.065 \mathrm{~m}$, the wave period $T_{w}=0.761 \mathrm{~s}$, the sinker weight $W_{s}=1.6 \mathrm{~kg}$.

modelled in the numerical model. Therefore, the numerical model simulated both the floater motion and net deformation via the CFD approach.

The numerical model was within the framework of OpenFOAM-3.0.x. In the numerical model, the floating fish cage system was simplified as a floater-net system. The mooring lines and the sinker were not resolved by the computational mesh. The numerical model couples a hydrodynamic solver for the net cage and the floater, the rigid body motion solver, the structural solver for the net cage, and the mesh motion solver in a segregated manner. The numerical model was carefully validated against three sets of cases in 2D. The first set of validation cases were for floater motion responses in regular waves. The second set of the validation cases were for the plane net panel without floater in steady current. The final validation cases were related to the whole floater-net system in regular waves and combined current and wave conditions.

Further work will be focused on the resolution of sinker and its motion by the computational mesh, which requires application of immersed boundary method or overset grid. Readers are referred to e.g. Kristiansen (2010) and Li et al. (2015) for application of these methods on marine hydrodynamic problems. Furthermore, extension of the numerical model to 3D cases is also an important part of the future work.

\section{Acknowledgement}

Dr. Mohsen Bardestani and Prof. Trygve Kristiansen from Norwegian University of Science and Technology is acknowledged for discussion with the first author on the physics of snap load. The research was partially supported by FP7-OCEAN-2011 project Innovative multi-purpose offshore platforms: planning, design and operation, MERMAID, 288710, under the call "Ocean of Tomorrow".

\section{References}

Bardestani, M., Faltinsen, O.M., 2013. A two dimensional approximation of a floating fish farm in waves and current with the effect of snap loads, in: Proceedings of the ASME 2013 32nd International Conference on Ocean, Offshore and Arctic Engineering, Nantes, France.

Bi, C., Zhao, Y., Dong, G., Zheng, Y., Gui, F., 2014a. A numerical analysis on the hydrodynamic characteristics of net cages using coupled fluid-structure interaction model. Aquacultural Engineering 59, 1-12. URL: http://linkinghub.elsevier. com/retrieve/pii/S014486091400003Xhttp://www.sciencedirect.com/science/article/pii/S014486091400003X. doi/10. 1016/j.aquaeng.2014.01.002

Bi, C.W., Zhao, Y.P., Dong, G.H., Xu, T.J., Gui, F.K., 2014b. Numerical simulation of the interaction between flow and flexible nets. Journal of Fluids and Structures 45, 180-201. URL: http://linkinghub.elsevier.com/retrieve/pii/ S0889974613002594 doi $10.1016 / \mathrm{j} \cdot \mathrm{jfluidstructs.2013.11.015}$ 
Birdsall, C.K., Langdon, A.B., 2004. Plasma physics via computer simulation. CRC Press.

Chen, H., Christensen, E., 2017. Development of a numerical model for fluid-structure interaction analysis of flow through and around an aquaculture net cage. Ocean Engineering 142. doi $10.1016 / \mathrm{j}$. oceaneng. 2017.07.033.

Chen, H., Christensen, E.D., 2016. Investigations on the porous resistance coefficients for fishing net structures. Journal of Fluids and Structures 65, 76-107. URL: http://www.sciencedirect.com/science/article/pii/S0889974615301687, doi 10. $1016 / j \cdot j$ fluidstructs.2016.05.005

Chow, J.H., Ng, E., 2016. Strongly coupled partitioned six degree-of-freedom rigid body motion solver with Aitken's dynamic under-relaxation. International Journal of Naval Architecture and Ocean Engineering 8, 320-329. URL: http://linkinghub. elsevier.com/retrieve/pii/S2092678216304393, doi 10.1016/j.ijnaoe.2016.04.001

Devolder, B., Schmitt, P., Rauwoens, P., Elsaesser, B., Troch, P., 2015. A review of the implicit motion solver algorithm in openfoam $(\mathbb{R}$ to simulate a heaving buoy, in: NUTTS conference 2015: 18th Numerical Towing Tank Symposium, pp. 1-6.

Dullweber, A., Leimkuhler, B., McLachlan, R., 1997. Symplectic splitting methods for rigid body molecular dynamics. The Journal of Chemical Physics 107, 5840. URL: http://scitation.aip.org/content/aip/journal/jcp/107/15/10.1063/1. 474310 doi $10.1063 / 1.474310$

Dunbar, A.J., Craven, B.A., Paterson, E.G., 2015. Development and validation of a tightly coupled CFD/6-DOF solver for simulating floating offshore wind turbine platforms. Ocean Engineering 110, 98-105. URL: http://dx.doi.org/10.1016/j. oceaneng.2015.08.066 doi $10.1016 / j$.oceaneng.2015.08.066

Faltinsen, O., 1993. Sea Loads on Ships and Offshore Structures. Cambridge Ocean Technology Series, Cambridge University Press. URL: https://books.google.dk/books?id=qZq4Rs2DZXoC

Fenton, J., 1988. The numerical solution of steady water wave problems. Computers \& Geosciences 14, 357-368. doi 10.1016/ 0098-3004(88)90066-0.

Förster, C., Wall, W.a., Ramm, E., 2007. Artificial added mass instabilities in sequential staggered coupling of nonlinear structures and incompressible viscous flows. Computer Methods in Applied Mechanics and Engineering 196, $1278-1293$. URL: http://linkinghub.elsevier.com/retrieve/pii/S0045782506002544 doi $10.1016 /$ j.cma.2006.09.002

Fu, S., Xu, Y., Hu, K., Zhong, Q., Li, R., 2014. Experimental Investigation on Hydrodynamics of a Fish Cage Floater-net System in Oscillatory and Steady Flows by Forced Oscillation Tests. Journal of Ship Research 58, 20-29. URL: http:// WWw. ingentaselect.com/rpsv/cgi-bin/cgi?ini=xref $\{\&\}$ body=linker $\{\&\} r e q d o i=10.5957 / J 0 S R .58 .1 .130022$, doi 10.5957/ JOSR.58.1.130022

Hirt, C., Nichols, B., 1981. Volume of fluid (VOF) method for the dynamics of free boundaries. Journal of Computational Physics 39, 201-225. URL: http://www.sciencedirect.com/science/article/pii/0021999181901455, doi 10.1016/0021-9991(81) 90145-5

Jacobsen, N.G., Fuhrman, D.R., Fredsoe, J., 2012. A wave generation toolbox for the open-source CFD library: OpenFoam (R). INTERNATIONAL JOURNAL FOR NUMERICAL METHODS IN FLUIDS 70, 1073-1088. doi 10.1002/fld.2726

James, D.F., Truong, Q.S., 1972. Wind load on cylinder with spanwise protrusion. Journal of the Engineering Mechanics Division 98, 1573-1589.

Jensen, B., Jacobsen, N.G., Christensen, E.D., 2014. Investigations on the porous media equations and resistance coefficients for coastal structures. Coastal Engineering 84, 56-72. URL: http://linkinghub.elsevier.com/retrieve/pii/ S0378383913001816 doi 10.1016/j.coastaleng.2013.11.004

Kristiansen, D., 2010. Wave Induced Effects on Floaters of Aquaculture plants. Ph.d. thesis. Norwegian University of Science and Technology.

Kristiansen, T., Faltinsen, O.M., 2012. Modelling of current loads on aquaculture net cages. Journal of Fluids and Structures 34, 218-235. URL: http://linkinghub.elsevier.com/retrieve/pii/S0889974612000783, doi 10.1016/j.jfluidstructs. 2012.04.001

Kristiansen, T., Faltinsen, O.M., 2015. Experimental and numerical study of an aquaculture net cage with floater in waves and current. Journal of Fluids and Structures 54, 1-26. URL: http://linkinghub.elsevier.com/retrieve/pii/ S0889974614002114 doi $10.1016 / \mathrm{j} . \mathrm{jfluidstructs.2014.08.015}$

Lader, P.F., Fredheim, A., 2006. Dynamic properties of a flexible net sheet in waves and current-A numerical approach. Aquacultural Engineering 35, 228-238. URL: http://linkinghub.elsevier.com/retrieve/pii/S0144860906000161, doi 10. $1016 / j$. aquaeng. 2006.02.002

Li, J., Castro, A.M., Carrica, P.M., 2015. A pressure-velocity coupling approach for high void fraction free surface bubbly flows in overset curvilinear grids. International Journal for Numerical Methods in Fluids 79, 343-369. doi 10.1002/fld.4054

Ma, L., Hu, K., Fu, S., Moan, T., Li, R., 2016. A Hybrid Empirical-Numerical Method for Hydroelastic Analysis of a Floater-and-Net System. Journal of Ship Research 60, 14-29. doi http://dx.doi.org/10.5957/JOSR.60.1.140058, arXiv:/dx.doi.org/10.5957/JOSR.60.1.140058

Newman, J.N., 1962. The Exciting Forces on Fixed Bodies in Waves. Journal of Ship Research, Society of Naval Architects and Marine Engineers 6, 1-10.

Newman, J.N., 1977. Marine hydrodynamics. MIT press.

Ong, M.C., Kamath, A., Bihs, H., Afzal, M.S., 2017. Numerical simulation of free-surface waves past two semi-submerged horizontal circular cylinders in tandem. Marine Structures 52, 1-14. URL: http://dx.doi.org/10.1016/j.marstruc.2016. 11.002 doi $10.1016 / j$.marstruc.2016.11.002

Palm, J., Eskilsson, C., Moura Paredes, G., Bergdahl, L., 2016. Coupled mooring analysis of floating wave energy converters using CFD. International Journal of Marine Energy 16, 83-99. URL: http://dx.doi.org/10.1016/j.ijome.2016.05.003, doi $10.1016 /$ j.ijome.2016.05.003

Patursson, Ø., Swift, M.R., Tsukrov, I., Simonsen, K., Baldwin, K., Fredriksson, D.W., Celikkol, B., 2010. Development of a porous media model with application to flow through and around a net panel. Ocean Engineering 37, 314-324. URL: 
Söding, H., 2001. How to integrate free motions of solids in fluids, in: 4th Numerical Towing Tank Symposium, Hamburg.

Xu, T.J., Dong, G.H., Li, Y.C., Guo, W.J., 2014. Numerical study of a self-submersible single-point mooring gravity cage in combined wave-current flow. Applied Ocean Research 48, 66-79. URL: http://dx.doi.org/10.1016/j.apor.2014.07.014 doi $10.1016 / j$. apor.2014.07.014

Xu, T.J., Zhao, Y.P., Dong, G.H., Gui, F.K., 2013. Analysis of hydrodynamic behavior of a submersible net cage and mooring system in waves and current. Applied Ocean Research 42, 155-167. URL: http://dx.doi.org/10.1016/j.apor.2013.05.007. doi $10.1016 / j$.apor.2013.05.007

Yao, Y., Chen, Y., Zhou, H., Yang, H., 2016. Numerical modeling of current loads on a net cage considering fluidstructure interaction. Journal of Fluids and Structures 62, 350-366. URL: http://linkinghub.elsevier.com/retrieve/pii/ S0889974616000116 doi $10.1016 / \mathrm{j} \cdot \mathrm{jfluidstructs.2016.01.004}$

Zhao, Y.P., Bai, X.D., Dong, G.H., Bi, C.W., Gui, F.K., 2015. Numerical analysis of the elastic response of a floating collar in waves. Ocean Engineering 95, 175-182. URL: http://linkinghub.elsevier.com/retrieve/pii/S0029801814004727. doi $10.1016 /$ j . oceaneng.2014.12.015

Zhao, Y.P., Bi, C.W., Dong, G.H., Gui, F.K., Cui, Y., Guan, C.T., Xu, T.J., 2013. Numerical simulation of the flow around fishing plane nets using the porous media model. Ocean Engineering 62, 25-37. URL: http://linkinghub.elsevier.com/ retrieve/pii/S0029801813000243 doi 10.1016/j.oceaneng.2013.01.009

Zhao, Y.P., Li, Y.C., Dong, G.H., Gui, F.K., Teng, B., 2007. A numerical study on dynamic properties of the gravity cage in combined wave-current flow. Ocean Engineering 34, 2350-2363. URL: http://linkinghub.elsevier.com/retrieve/pii/ S0029801807001400 doi $10.1016 / j$. oceaneng.2007.05.003 